\title{
Interaction between phase transformations and dislocations at the nanoscale. Part 2. Phase field simulation examples
}

\author{
Mahdi Javanbakht ${ }^{a, b}$ and Valery I. Levitas ${ }^{c, *}$ \\ ${ }^{a}$ Isfahan University of Technology, Department of Mechanical Engineering, Isfahan, Iran, \\ ${ }^{b}$ Iowa State University, Department of Aerospace Engineering, Ames, Iowa 50011, USA, \\ ${ }^{c}$ Iowa State University, Departments of Aerospace Engineering, Mechanical Engineering, and \\ Material Science and Engineering, Ames, Iowa 50011, USA.
}

\begin{abstract}
The complete system of phase field equations for coupled martensitic phase transformations (PTs), dislocation evolution, and mechanics at large strains is presented. Finite element method (FEM) is utilized to solve this system for two important problems. The first one is related to the simulation of shear strain-induced PT at the evolving dislocation pile-ups in a nanosized bicrystal. Plasticity plays a dual part in the interaction with PT. Dislocation pile-ups produce strong stress tensor concentrators that lead to barrierless martensite (M) nucleation. On the other hand, plasticity in the transforming grain relaxes these stress concentrators suppressing PT. The final stationary M morphology is governed by the local thermodynamic equilibrium, either at the interfaces or in terms of stresses averaged over the martensitic region or the entire grain. This is very surprising because of strong heterogeneity of stress fields and is in contrast to previous statements that phase equilibrium conditions do not enter the description of strain-induced PTs. The second problem is devoted to martensitic plate propagation through a bicrystal during temperature-induced PT. For elastic growth (without dislocations) and a large thermal driving force, a complex transformation path with plate branching and direct and reverse PTs is observed, which still ends with the same stationary nanostructure as for a smaller driving force and a traditional transformation path. Sharp grain boundary arrests plate growth at a relatively small driving force, exhibiting an athermal friction. For elastoplastic growth, the generation of dislocations produces athermal friction and arrests the plate below some critical driving force, leading to a morphological transition from plate to lath $\mathrm{M}$. The width of the martensitic plate increases in comparison with elastic growth
\end{abstract}

\footnotetext{
* Corresponding author. Email address: vlevitas@iastate.edu, tel. (515)-294-9691 (V. I. Levitas)
} 
due to internal stress relaxation. Plate growth is accompanied by the nucleation of dislocations within $\mathrm{M}$ and remaining in $\mathrm{M}$, the nucleation of dislocations at the tip of a plate and spreading them in austenite (A), and passing some dislocations through $\mathrm{M}$, then through a M-A interface, and then through $A$. Due to the existence of a stationary equilibrium $M$ nanostructure and concentration for each temperature, for a large enough observation time one observes athermal, rate- and time-independent kinetics, even while local kinetics is rate dependent. In the final structure, most dislocations are in $\mathrm{M}$ despite its having a yield strength three times larger than for $A$, which is consistent with experiments.

Keywords: Phase field approach; Phase transformation; Dislocations; Large strains; Inheritance of dislocations; Finite element simulations.

\section{Introduction}

Since quite detailed review of the literature on the interaction between martensitic PTs and plasticity was given in Part 1 of this paper (Levitas and Javanbakht (2015)), we will mention here some additional aspects related to numerical solutions only. Some reviews on earlier works on the topic have been published in Olson and Cohen (1986); Fischer et al. (1994); Olson and Roytburd (1995); Fischer et al. (1996); Levitas (2004a); Lovey and Torra (1999). At the microscale, various sharp-interface theories for martensitic PTs coupled with classical continuum plasticity have been utilized for FEM solutions of various problems on the appearance of a $\mathrm{M}$ region, interface propagation, and microstructure evolution in Marketz and Fischer (1994, 1995); Cherkaoui et al (1998); Cherkaoui and Berveiller (2000); Fischer and Reisner (1998); Fischer et al. (2000); Idesman et al. (1998). FEM solutions for a semi-coherent interface in an elastoplastic material were presented in Idesman et al. (1997, 1998, 1999, 2000). Finite strain numerical solutions to various problems were found in Idesman et al. (1999); Levitas et al. (1999, 2002); Idesman et al. (2000). Micromechanics-based formulation and FEM solutions are presented in Turteltaub and Suiker (2005); Kouznetsova and Geers (2008). Macroscopic simulation of the interaction between PT and plasticity in different samples, based on macroscopic (averaged) constitutive equations was presented in Levitas and Zarechnyy (2010a,b); Sitko and Skoczen (2012); Mahnken et al. (2012). Discrete dislocation plasticity combined with the nucleation and growth of elliptic $\mathrm{M}$ plates with the prescribed aspect ratio 
have been presented in Shi et al. (2010).

The main focus of the current paper is on phase field approach (PFA) for the interaction between evolving martensitic PTs and discrete dislocations. For stationary dislocations, which were introduced through their stationary stress field, the nucleation of a $\mathrm{M}$ region was studied numerically in Reid et al. (1998); Wang and Khachaturyan (2006). M evolution with dislocations located at the moving phase interface have been treated by Kundin et al. (2011a). PFA solutions for martensitic PT with continuum dislocation theory are presented in Kundin et al. (2011b) and with macroscopic von Mises plasticity in Cottura et al. (2012); Yeddu et al. (2012a); Malik et al. (2012); Yamanaka et al. (2008).

An advanced PFA for martensitic PTs and dislocation evolution at large strains has been developed in Levitas and Javanbakht (2015). It combines the most advanced and the only available large-strain PFA for multivariant martensitic PTs (Levitas (2013b)) and dislocation evolutions (Levitas and Javanbakht $(2012,2014 b))$ with their nontrivial interactions. Both theories for PTs (Levitas (2013b)) and dislocations (Levitas and Javanbakht (2012, 2014b)) satisfy some important requirements related to equilibrium and instability conditions formulated in Levitas and Preston $(2002 \mathrm{a}, \mathrm{b})$ in order to describe typical features of stress-strain curves that are conceptually consistent with known experimental data, guarantee constant (stress- and temperature-independent) transformation deformation gradient and Burgers vector, and allow one to include all thermomechanical properties of both $\mathrm{A}$ and $\mathrm{M}$ variants. In addition, PFA for dislocation evolution in Levitas and Javanbakht (2012, 2014b) defines dislocation height by equations rather than by computational mesh in previous approaches (Wang et al. (2001a,b); $\mathrm{Hu}$ and Chen $(2001,2002))$. The interaction between PT and dislocations includes: (a) a multiplicative kinematic decomposition of the deformation gradient into elastic, transformational, and plastic parts; (b) the inheritance of dislocations of $A$ in $M$ during martensitic PT and dislocations of $\mathrm{M}$ in $\mathrm{A}$ during reverse PT, as well as their further evolution along the nontraditional slip systems; (c) dependence of all material parameters for dislocations on the order parameters that describe PT and additional contributions to the driving force for PT due to this dependence. The main interaction between dislocations and PT occurs through stress fields generated by their eigen strains and is determined by a solution of coupled PFA and mechanical problems. 
In Levitas and Javanbakht (2012, 2013, 2014a), solutions to some material problems on coupling between martensitic PT and dislocation evolution have been obtained utilizing a simplified version of the PFA. These solutions allowed us to elucidate some important effects, such as scale-dependent athermal hysteresis for the semicoherent interface motion and mechanism of semicoherent interface motion, pushing and inheriting dislocations by propagating phase interface, and the significant reduction in PT pressure due to a dislocation pile-up generated by applied shear stresses.

In the current paper, general PFA for the interaction between PT and dislocation evolution developed in Levitas and Javanbakht (2015) is applied for FEM simulations of two important problems, which are analyzed in detail. The paper is organized as follows. In Section 2 a complete system of the geometrically nonlinear equations for coupled PFA for single-variant PTs, dislocation evolution, and continuum mechanics is formulated in the reference configuration and also simplified for geometrically linear approximation. Material parameters and numerical method are presented. In Section 3 the interaction between PTs and dislocations in a nanograin material is analyzed under plastic shear. In Section 4, the temperature-induced nucleation, growth, and arrest of $\mathrm{M}$ plate in an A bicrystal is studied. Various nontrivial effects are found for both problems. Section 5 contains concluding remarks.

\section{Complete system of equations}

For simplicity, we will focus on the particular case of coinciding slip systems of A and transformed back $\mathrm{M}$. This is true for PTs between body centric cubic and body centric tetragonal lattices as well as for PTs between face centric cubic and face centric tetragonal lattices considered in the examples below, see 2D sketch in Fig. 1. In this case just a single set of the order parameters $\xi_{\alpha}$, which characterizes slip along any slip systems of $\mathrm{A}$ and $\mathrm{M}$, is sufficient to describe dislocations independent of whether it occurs in A or M. Also, we will limit ourselves by a single $\mathrm{M}$ variant, mechanical equilibrium without body forces, and surface energy, which is independent of phase and dislocations. Then the complete system of equations formulated in Levitas and Javanbakht (2015) is presented in Box. 1. For convenience, we keep the same structure of the Box like in Levitas and Javanbakht (2015). Also, for those who needs to solve similar problems for infinitesimal strains, simplified small strain version is presented as well. 

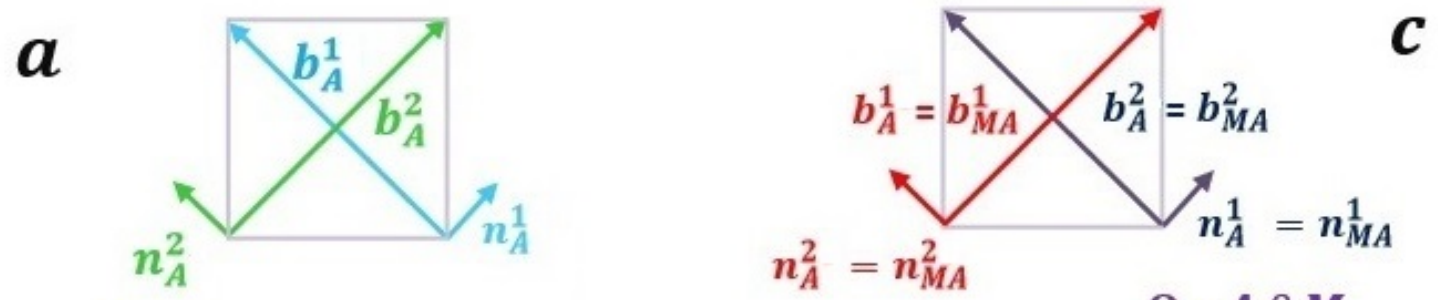

$\Omega_{0}, A$
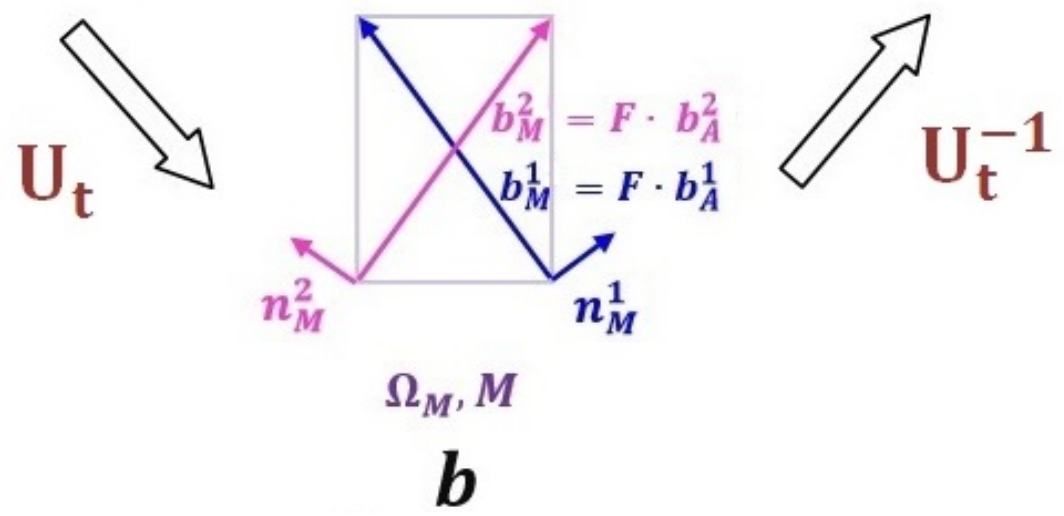

Figure 1: (color online). Schematics for Burgers vectors and normals to the slip planes in austenite and martensite in different configurations for the case when the slip system of martensite $\left(\boldsymbol{b}_{M}^{\omega}, \boldsymbol{n}_{M}^{\omega}\right)$ coincides with the transformed slip systems of austenite $\left(\boldsymbol{b}_{A M}^{\alpha}=\boldsymbol{U}_{t} \cdot \boldsymbol{b}_{A}^{\alpha}, \boldsymbol{n}_{A M}^{\alpha}=\boldsymbol{n}_{A}^{\alpha} \cdot \boldsymbol{U}_{t}^{-1} /\left|\boldsymbol{n}_{A}^{\alpha} \cdot \boldsymbol{U}_{t}^{-1}\right|\right)$. (a) Two dimensional body (or face) centric cubic lattice of austenite with two slip systems $\left(\boldsymbol{b}_{A}^{\alpha}, \boldsymbol{n}_{A}^{\alpha}\right)$ along the diagonals in the initial configuration $\Omega_{0}$. (b) Two dimensional body (or face) centric tetragonal lattice of martensite with two slip systems $\left(\boldsymbol{b}_{M}^{\alpha}, \boldsymbol{n}_{M}^{\alpha}\right)$ along the diagonals in the transformed configuration $\Omega_{t}$, which coincide with slip systems of austenite inherited by martensite $\left(\boldsymbol{b}_{A M}^{\alpha}, \boldsymbol{n}_{A M}^{\alpha}\right)$. (c) Slip systems of martensite inherited by austenite $\left(\boldsymbol{b}_{M A}^{\alpha}\right.$, $\boldsymbol{n}_{M A}^{\alpha}$ ) during reverse phase transformations in the reference configuration $\Omega_{0}$ coincide with the slip systems of austenite in austenite $\left(\boldsymbol{b}_{A}^{\alpha}, \boldsymbol{n}_{A}^{\alpha}\right)$.

\section{Box 1.}

\section{Kinematics}

I. Large strains

1.1. Multiplicative decomposition of the deformation gradient $\boldsymbol{F}$ into elastic $\boldsymbol{F}_{e}$, transformation $\boldsymbol{U}_{t}$, and plastic $\boldsymbol{F}_{p}$ parts

$$
\boldsymbol{F}=\boldsymbol{F}_{e} \cdot \boldsymbol{U}_{t} \cdot \boldsymbol{F}_{p}
$$


1.2. Jacobian determinants

$$
\begin{aligned}
& J:=\frac{d V}{d V_{0}}=\frac{\rho_{0}}{\rho}=\operatorname{det} \boldsymbol{F} ; \quad J_{e}:=\frac{d V}{d V_{t}}=\frac{\rho_{t}}{\rho_{0}}=\operatorname{det} \boldsymbol{F}_{e} ; \\
& J_{t p}:=\frac{d V_{t}}{d V_{p}}=\frac{\rho_{p}}{\rho_{t}}=\operatorname{det} \boldsymbol{U}_{t} \operatorname{det} \boldsymbol{F}_{p}=\operatorname{det} \boldsymbol{U}_{t}=J_{t} ; \quad J_{p}:=\operatorname{det} \boldsymbol{F}_{p}=1 ; \quad J=J_{e} J_{t},
\end{aligned}
$$

where $V_{0}\left(\rho_{0}\right), V_{t}\left(\rho_{t}\right), V_{p}\left(\rho_{p}\right)$, and $V(\rho)$ are the volumes (mass densities) in the reference $\left(\Omega_{0}\right)$, intermediate $\left(\Omega_{t}\right.$, after elastic unloading), intermediate $\left(\Omega_{p}\right.$, after elastic unloading and reverse $\mathrm{PT})$, and actual $(\Omega)$ configurations, respectively.

1.3. Transformation-deformation gradient

$$
\boldsymbol{U}_{t}=\boldsymbol{I}+\bar{\varepsilon}_{t} \varphi(a, \eta) ; \quad \varphi(a, \eta)=a \eta_{k}^{2}(1-\eta)^{2}+\left(4 \eta^{3}-3 \eta^{4}\right) ; \quad 0<a<6
$$

where $\overline{\boldsymbol{\varepsilon}}_{t}$ is the transformation strain of a $\mathrm{M}$ variant under study and $\eta$ is the order parameter that describes phase transformation from $\mathrm{A}(\eta=0)$ to $\mathrm{M}(\eta=1)$.

1.4. Plastic part of the velocity gradient

$$
\begin{aligned}
& \boldsymbol{l}_{p}:=\sum_{\alpha=1}^{p} \frac{1}{H^{\alpha}} \boldsymbol{b}^{\alpha} \otimes \boldsymbol{n}^{\alpha} \dot{\Phi}\left(\xi_{\alpha}\right)=\sum_{\alpha=1}^{p} \gamma_{\alpha} \boldsymbol{m}^{\alpha} \otimes \boldsymbol{n}^{\alpha} \dot{\Phi}\left(\xi_{\alpha}\right), \\
& \Phi\left(\xi_{\alpha}\right)=\phi\left(\bar{\xi}_{\alpha}\right)+\operatorname{Int}\left(\xi_{\alpha}\right) ; \quad \phi\left(\bar{\xi}_{\alpha}\right)=\bar{\xi}_{\alpha}^{2}\left(3-2 \bar{\xi}_{\alpha}\right),
\end{aligned}
$$

where $\boldsymbol{b}^{\alpha}$ is the Burgers vector of a dislocation in the $\alpha^{t h}$ slip system, $\boldsymbol{n}^{\alpha}$ is the unit normal to the slip plane; $\gamma_{\alpha}=\left|\boldsymbol{b}^{\alpha}\right| / H^{\alpha}$ is the plastic shear strain per single dislocation in a dislocation band of the height $H^{\alpha}, \boldsymbol{m}^{\alpha}$ is the unit vector in the direction of $\boldsymbol{b}^{\alpha}, \xi_{\alpha}$ is the order parameter for a dislocation in the $\alpha^{t h}$ slip system, and $\operatorname{Int}\left(\xi_{\alpha}\right)$ and $\bar{\xi}_{\alpha}:=\xi_{\alpha}-\operatorname{Int}\left(\xi_{\alpha}\right)$ is the integer and fractional parts of $\xi_{\alpha}$.

II. Small strains

$$
\begin{aligned}
& \boldsymbol{\varepsilon}=(\boldsymbol{\nabla} \boldsymbol{u})_{s}=\boldsymbol{\varepsilon}_{e}+\boldsymbol{\varepsilon}_{t}+\boldsymbol{\varepsilon}_{p} ; \quad \boldsymbol{\varepsilon}_{t}=\overline{\boldsymbol{\varepsilon}}_{t} \varphi\left(a, \eta_{k}\right) \\
& \boldsymbol{\omega}=\boldsymbol{\omega}_{e}+\boldsymbol{\omega}_{t}+\boldsymbol{\omega}_{p} ; \quad \boldsymbol{\varepsilon}_{p}+\boldsymbol{\omega}_{p}=\sum_{\alpha=1}^{p} \frac{1}{H^{\alpha}} \boldsymbol{b}^{\alpha} \otimes \boldsymbol{n}^{\alpha} \Phi\left(\xi_{\alpha}\right),
\end{aligned}
$$

where $\boldsymbol{u}$ is the displacement; $\boldsymbol{\nabla}$ is the gradient operator (in the reference configuration); subscript $s$ stands for symmetrization, $\boldsymbol{\varepsilon}$ and $\boldsymbol{\omega}$ are the small strain and rotations, respectively; subscripts $e, t$, and $p$ stand for elastic, transformational, and plastic parts, respectively.

\section{Helmholtz free energy per unit mass}

$$
\psi=J_{t} \psi^{e}+\psi_{\eta}^{\theta}+\psi_{\xi}^{c}+\psi_{\xi}^{i n t}+\psi_{\eta}^{\nabla}+\psi_{\xi}^{\nabla} .
$$




\subsection{Elastic energy}

$$
\rho_{0} \psi^{e}=\frac{1}{2} \boldsymbol{E}_{e}: \boldsymbol{C}: \boldsymbol{E}_{e}
$$

where $\boldsymbol{E}_{e}=0.5\left(\boldsymbol{F}_{e}^{T} \cdot \boldsymbol{F}_{e}-\boldsymbol{I}\right)$ is the elastic Lagrangian strain measure tensor and $\boldsymbol{C}$ is the tensor of elastic moduli, which is assumed to be phase-independent.

\subsection{Thermal energy}

$$
\begin{aligned}
& \psi_{\eta}^{\theta}=A \eta^{2}(1-\eta)^{2}+\Delta G^{\theta}\left(4 \eta^{3}-3 \eta^{4}\right) \\
& \Delta G^{\theta}=-\Delta s\left(\theta-\theta_{e}\right), \quad A=A_{0}\left(\theta-\theta_{c}\right), \quad A_{0}>0
\end{aligned}
$$

where $\Delta G^{\theta}$ and $\Delta s$ are the differences between the thermal part of the free energy and entropy for $\mathrm{M}$ and $\mathrm{A}$, respectively; $A$ is the double-well barrier between $\mathrm{A}$ and $\mathrm{M} ; \theta_{e}$ is the equilibrium temperature for stress-free $\mathrm{A}$ and $\mathrm{M} ; A_{0}$ is a parameter, and $\theta_{c}$ is the critical temperature at which stress-free A loses its thermodynamic stability.

2.3. Crystalline energy

$$
\begin{aligned}
& \psi_{\xi}^{c}=\sum_{\alpha=1}^{p} A_{\alpha}\left(\eta, \bar{y}^{\alpha}\right)\left(\bar{\xi}_{\alpha}\right)^{2}\left(1-\bar{\xi}_{\alpha}\right)^{2} ; \\
& A_{\alpha}^{A, M}\left(y^{\alpha}\right)=\left\{\begin{array}{ll}
\bar{A}_{\alpha}^{A, M} & \bar{A}_{\alpha}\left(\eta, \bar{y}^{\alpha}\right)=A_{\alpha}^{A}+\left(A_{\alpha}^{M}-A_{\alpha}^{A}\right) \eta^{2}(3-2 \eta) \\
k A_{\alpha}^{A, M} & \bar{y}^{\alpha}>H^{\alpha}
\end{array} \quad \bar{y}^{\alpha}=y^{\alpha}-\operatorname{Int}\left(\frac{y^{\alpha}}{H^{\alpha}+w_{\alpha}}\right)\left(H^{\alpha}+w_{\alpha}\right),\right.
\end{aligned}
$$

where $A_{\alpha}^{A}$ and $A_{\alpha}^{M}$ are the magnitudes of the multi-well crystalline energy in $\mathrm{A}$ and $\mathrm{M}$, respectively, which determine the critical shear stress for barrierless nucleation of dislocation (theoretical strength in shear); $y^{\alpha}$ is the coordinate normal to the $\alpha_{t h}$ slip plane; $w_{\alpha}$ is the width of the thin layer between dislocation bands.

2.4. Energy of interaction of dislocation cores belonging to different slip systems

$$
\begin{aligned}
& \psi_{\xi}^{i n t}=\sum_{\alpha, k=1}^{p} A_{\alpha k}(\eta)\left(\bar{\xi}_{\alpha}\right)^{2}\left(1-\bar{\xi}_{\alpha}\right)^{2}\left(\bar{\xi}_{k}\right)^{2}\left(1-\bar{\xi}_{k}\right)^{2} ; \quad A_{\alpha \alpha}=0 \\
& A_{\alpha k}(\eta)=A_{\alpha k}^{A}+\left(A_{\alpha k}^{M}-A_{\alpha k}^{A}\right) \eta^{2}(3-2 \eta),
\end{aligned}
$$

where $A_{\alpha k}^{A}$ and $A_{\alpha k}^{M}$ are the coefficients of the energy of interaction of dislocation cores in $\mathrm{A}$ and $\mathrm{M}$, respectively.

2.5. Gradient energies for PTs and dislocations

$$
\begin{aligned}
& \psi_{\eta}^{\nabla}=\frac{\beta^{\eta}}{2}|\nabla \eta|^{2} ; \quad \psi_{\xi}^{\nabla}=0.5 \beta_{\xi}(\eta) \sum_{\alpha=1}^{p}\left(\left(\nabla^{m} \xi_{\alpha}\right)^{2}+Z\left(1-\bar{\xi}_{\alpha}\right)^{2}\left(\nabla^{n} \xi_{\alpha}\right)^{2}\right) ; \\
& \beta_{\xi}(\eta)=\beta_{\xi}^{A}+\left(\beta_{\xi}^{M}-\beta_{\xi}^{A}\right) \eta^{2}(3-2 \eta) ; \quad \nabla^{m} \xi_{\alpha}:=\nabla \xi_{\alpha} \cdot \boldsymbol{m}^{\alpha} ; \quad \nabla^{n} \xi_{\alpha}:=\nabla \xi_{\alpha} \cdot \boldsymbol{n}^{\alpha},
\end{aligned}
$$


where $\beta^{\eta}$ is the coefficient of the gradient energy for PT; $\beta_{\xi}^{A}$ and $\beta_{\xi}^{M}$ are the coefficient of the gradient energy for dislocations in $\mathrm{A}$ and $\mathrm{M}$, respectively; $Z$ is the ratio of the coefficients for the gradient energy normal to and along the slip plane; superscripts $m$ and $n$ stand for the directions along and the normal to the slip plane, respectively.

\section{First Piola-Kirchhoff $P$ and Cauchy $\sigma$ stress tensor}

I. Large strains

$$
\begin{gathered}
\boldsymbol{P}=\rho_{0} J_{t} \boldsymbol{F}_{e} \cdot \frac{\partial \psi^{e}}{\partial \boldsymbol{E}_{e}} \cdot \boldsymbol{U}_{t}^{-1} \cdot \boldsymbol{F}_{p}^{T-1}=J_{t} \boldsymbol{F}_{e} \cdot \boldsymbol{C}: \boldsymbol{E}_{e} \cdot \boldsymbol{U}_{t}^{-1} \cdot \boldsymbol{F}_{p}^{T-1} \\
\boldsymbol{\sigma}=\rho J_{t} \boldsymbol{F}_{e} \cdot \frac{\partial \psi^{e}}{\partial \boldsymbol{E}_{e}} \cdot \boldsymbol{F}_{e}^{T}=\frac{1}{J_{e}} \boldsymbol{F}_{e} \cdot \boldsymbol{C}: \boldsymbol{E}_{e} \cdot \boldsymbol{F}_{e}^{T}
\end{gathered}
$$

II. Small strains and linear elasticity

$$
\boldsymbol{\sigma}=\rho \frac{\partial \psi}{\partial \boldsymbol{\varepsilon}_{e}}=\boldsymbol{C}: \varepsilon_{e}
$$

\section{Ginzburg-Landau equations}

4.1. Compact form in the reference configuration at large strains

$$
\begin{aligned}
& \dot{\eta}=L^{\eta} X^{\eta}=L^{\eta}\left(\frac{1}{\rho_{0}} \boldsymbol{P}^{T} \cdot \boldsymbol{F}_{e}: \frac{\partial \boldsymbol{U}_{t}}{\partial \eta} \cdot \boldsymbol{F}_{p}+\boldsymbol{\nabla} \cdot\left(\frac{\partial \psi}{\partial \boldsymbol{\nabla} \eta}\right)-\frac{\partial \psi}{\partial \eta}\right) \\
& \dot{\xi}_{\alpha}=L_{\alpha}(\eta) X_{\alpha}^{\xi}=L_{\alpha}(\eta)\left(\frac{1}{\rho_{0}} \tau_{\alpha} \gamma_{\alpha} \frac{\partial \Phi}{\partial \xi_{\alpha}}+\boldsymbol{\nabla} \cdot\left(\frac{\partial \psi}{\partial \boldsymbol{\nabla} \xi_{\alpha}}\right)-\frac{\partial \psi}{\partial \xi_{\alpha}}\right) ; \\
& L_{\alpha}(\eta)=L_{\alpha}^{A}+\left(L_{\alpha}^{M}-L_{\alpha}^{A}\right) \eta^{2}(3-2 \eta) ; \quad \tau_{\alpha}:=\boldsymbol{n}^{\alpha} \cdot \boldsymbol{F}_{p} \cdot \boldsymbol{P}^{T} \cdot \boldsymbol{F}_{e} \cdot \boldsymbol{U}_{t} \cdot \boldsymbol{m}^{\alpha},
\end{aligned}
$$

where $L^{\eta}$ is the kinetics coefficient for PT; $X^{\eta}$ is the dissipative force conjugate to $\dot{\eta}$; $X^{\xi}$ is the dissipative force conjugate to $\dot{\xi}_{\alpha} ; L_{\alpha}^{A}$ and $L_{\alpha}^{M}$ are the kinetics coefficients for dislocations in $\mathrm{A}$ and $\mathrm{M}$, respectively; $\tau_{\alpha}$ is the resolved shear stress for a dislocation.

4.2. Detailed form at large strains

$$
\begin{aligned}
& \dot{\eta}=L^{\eta}\left\{\left(\frac{1}{\rho_{0}} \boldsymbol{P}^{T} \cdot \boldsymbol{F}_{e}: \frac{\partial \boldsymbol{U}_{t}}{\partial \eta} \cdot \boldsymbol{F}_{p}-J_{t} \boldsymbol{U}_{t}^{-1}: \frac{\partial \boldsymbol{U}_{t}}{\partial \eta} \psi^{e}\left(\boldsymbol{E}_{e}, \eta\right)-J_{t} \frac{\partial \psi^{e}\left(\boldsymbol{E}_{e}, \eta\right)}{\partial \eta}+\right.\right. \\
& -\left[2 A \eta(1-\eta)(1-2 \eta)+12 \Delta G^{\theta} \eta^{2}(1-\eta)\right]-\sum_{\alpha=1}^{p} \frac{\partial A_{\alpha}\left(\eta, \bar{y}^{\alpha}\right)}{\partial \eta}\left(\bar{\xi}_{\alpha}\right)^{2}\left(1-\bar{\xi}_{\alpha}\right)^{2}- \\
& \sum_{\alpha, k=1}^{p} \frac{\partial A_{\alpha k}(\eta)}{\partial \eta}\left(\bar{\xi}_{\alpha}\right)^{2}\left(1-\bar{\xi}_{\alpha}\right)^{2}\left(\bar{\xi}_{k}\right)^{2}\left(1-\bar{\xi}_{k}\right)^{2} \\
& \left.-0.5 \frac{\partial \beta_{\xi}(\eta)}{\partial \eta} \sum_{\alpha=1}^{p}\left(\left(\nabla^{m} \xi_{\alpha}\right)^{2}+Z\left(1-\bar{\xi}_{\alpha}\right)^{2}\left(\nabla^{n} \xi_{\alpha}\right)^{2}\right)+\beta^{\eta} \nabla^{2} \eta\right\}
\end{aligned}
$$




$$
\begin{aligned}
& \dot{\xi}_{\alpha}=L_{\alpha}(\eta)\left\{\frac{6}{\rho_{0}} \tau_{\alpha} \gamma_{\alpha} \bar{\xi}_{\alpha}\left(1-\bar{\xi}_{\alpha}\right)+\frac{1}{2} \boldsymbol{\nabla} \beta_{\xi}(\eta) \cdot \nabla \bar{\xi}_{\alpha}+\frac{1}{2}\left[Z\left(1-\bar{\xi}_{\alpha}\right)^{2}-1\right]\left(\nabla \bar{\xi}_{\alpha} \cdot \boldsymbol{n}^{\alpha}\right)\left(\nabla \beta_{\xi}(\eta) \cdot \boldsymbol{n}^{\alpha}\right)\right. \\
& +\frac{1}{2} \beta_{\xi}(\eta)\left[\nabla^{2} \bar{\xi}_{\alpha}+\left(Z\left(1-\bar{\xi}_{\alpha}\right)^{2}-1\right)\left(\boldsymbol{\nabla} \cdot \boldsymbol{n}^{\alpha}\right)\left(\boldsymbol{\nabla} \bar{\xi}_{\alpha} \cdot \boldsymbol{n}^{\alpha}\right)\right] \\
& -2 Z\left(1-\bar{\xi}_{\alpha}\right)\left(\nabla \bar{\xi}_{\alpha} \cdot \boldsymbol{n}^{\alpha}\right)^{2}+\left[Z\left(1-\bar{\xi}_{\alpha}\right)^{2}-1\right] \nabla\left(\nabla \bar{\xi}_{\alpha} \cdot \boldsymbol{n}^{\alpha}\right) \cdot \boldsymbol{n}^{\alpha}-2 A_{\alpha}\left(\eta, \bar{y}^{\alpha}\right) \bar{\xi}_{\alpha}\left(1-\bar{\xi}_{\alpha}\right)\left(1-2 \bar{\xi}_{\alpha}\right) \\
& \left.-2 A_{\alpha k}(\eta) \bar{\xi}_{\alpha}\left(1-\bar{\xi}_{\alpha}\right)\left(1-2 \bar{\xi}_{\alpha}\right)\left(\bar{\xi}_{k}\right)^{2}\left(1-\bar{\xi}_{k}\right)^{2}+\beta_{\xi}(\eta) Z\left(1-\bar{\xi}_{\alpha}\right)\left(\nabla^{n} \xi_{\alpha}\right)^{2}\right\} .
\end{aligned}
$$

4.3. Small strains, linear elasticity

$$
\begin{aligned}
& \dot{\eta}=L_{\eta}\left\{\left(\frac{1}{\rho_{0}} \boldsymbol{\sigma}: \frac{\partial \boldsymbol{\varepsilon}_{t}}{\partial \eta}-\frac{J_{t}}{2 \rho_{0}}\left(\boldsymbol{I}: \frac{\partial \boldsymbol{\varepsilon}_{t}}{\partial \eta}\right) \boldsymbol{\varepsilon}_{e}: \boldsymbol{C}: \boldsymbol{\varepsilon}_{e}\right.\right. \\
& -\left[2 A \eta(1-\eta)(1-2 \eta)+12 \Delta G^{\theta} \eta^{2}(1-\eta)\right]-\sum_{\alpha=1}^{p} \frac{\partial A_{\alpha}\left(\eta, \bar{y}^{\alpha}\right)}{\partial \eta}\left(\bar{\xi}_{\alpha}\right)^{2}\left(1-\bar{\xi}_{\alpha}\right)^{2}- \\
& \sum_{\alpha, k=1}^{p} \frac{\partial A_{\alpha k}(\eta)}{\partial \eta}\left(\bar{\xi}_{\alpha}\right)^{2}\left(1-\bar{\xi}_{\alpha}\right)^{2}\left(\bar{\xi}_{k}\right)^{2}\left(1-\bar{\xi}_{k}\right)^{2} \\
& \left.-0.5 \frac{\partial \beta_{\xi}(\eta)}{\partial \eta} \sum_{\alpha=1}^{p}\left(\left(\nabla^{m} \xi_{\alpha}\right)^{2}+Z\left(1-\bar{\xi}_{\alpha}\right)^{2}\left(\nabla^{n} \xi_{\alpha}\right)^{2}\right)+\beta^{\eta} \nabla^{2} \eta\right\} .
\end{aligned}
$$

For dislocations, the Ginzburg-Landau equation for small distortions is the same as Eq.(18).

\section{Equilibrium equation}

$$
\nabla \cdot P=0 ; \quad \nabla \cdot \sigma=0
$$

\section{Boundary conditions for the order parameters}

$$
\boldsymbol{n}_{0} \cdot \nabla \eta=0, \quad \nabla \bar{\xi}_{\alpha} \cdot \boldsymbol{b}^{\alpha}=0
$$

where $\boldsymbol{n}_{0}$ is the normal to the surface in $\Omega_{0}$.

Numerical solutions. FEM and the code COMSOL Multiphysics code have been utilized. Plane strain problems and straight edge dislocations are considered below. Cubic to tetragonal PT is studied. Slip directions in cubic lattice are oriented at $60^{0}$ to each other, which is usually accepted in 2D simulations (Asaro (1983); Shi et al. (2011)). Boundary conditions Eq.(21) for the order parameters assume no change in the surface energy during PT and when

dislocations exit at the surface. The isotropic tensor of elastic moduli is used in Eq.(13) with shear modulus $\mu=71.5 \mathrm{GPa}$ and bulk modulus $K=112.6 \mathrm{GPa}$. Parameters in the periodic 
crystalline energy of dislocations $\psi_{\xi}^{c}$ (Eq.(9)) are chosen as $k=100$ and $w_{\alpha}=0.1 H^{\alpha}$. Due to large geometric changes remeshing is required to eliminate large deformation and inversion of mesh, which leads to divergence of the solution. Embedded in COMSOL procedure applies remeshing automatically at some time steps during solution. There is possibility to select the time range during which remeshing is allowed and controlling the range of mesh sizes. Remeshing is produced in local regions only where elements are drastically distorted, mainly near stress concentrators, as well when different types of meshes in different regions are utilized. New type of mesh may also be generated automatically.

Several COMSOL's moduli have been utilized for solution of the formulated system of equations; the most general finite strain case requires application of PDE (partial differential equations) modulus. The backward time differentiation scheme is utilized for solution of the non-stationary coupled problems. Quadrilateral finite elements with the quadratic polynomial for the shape function is utilized unless stated otherwise. This leads to reduction of number of the degrees of freedom and the computation time in comparison with linear finite elements. Quadrilateral finite elements also improve convergence. In some problems, the fifth degree polynomial for the shape function reduces significantly stress oscillations between dislocation band and the rest of a crystal.

Numerical solution converges to an analytical solution for a propagating phase interface for homogeneous applied stresses (Levitas et al. (2010)) for 4-5 elements per interface width (Levitas and Javanbakht (2011a)). It converges to a similar analytical solution for a dislocation (Lee et al. (2011)) for 7 finite elements per dislocation height and 6 elements per width of a core. Such a discretization has been used in our simulations. Analytical solutions tend to a sharp interface and dislocation when $\beta^{\eta} \rightarrow 0$ and $\beta_{\xi} \rightarrow 0$, respectively. A time step of $1^{-5} p s$ is used in our simulations. Our code can reproduce known crystallographic solutions for an invariant-plane phase interface for large strain and rotations as well as experimentally observed sophisticated microstructure with twin bending and spitting (Levitas et al. (2013)). Analytical solution for a spacing between incoherency dislocations is reproduced in Levitas and Javanbakht (2012). Numerical solution for nucleation at dislocation pile-up in Levitas and Javanbakht (2014a) at initial stage reproduces well analytical solution in Levitas (2004b). Our numerous simulations of the PT and dislocation problems mentioned in Introduction produce 
reasonable results, which gives a hope that solutions for interaction of $\mathrm{PT}$ and dislocations are sufficiently accurate as well. Typical computation time for problems in Section 3 is 16 hours and in Section 4 is 48 hours, both using eight core personal computer.

All size, stress, and time parameters are normalized by $1 \mathrm{~nm}, 1 \mathrm{GPa}$, and $1 p s$, respectively. While simulations are performed in the reference configuration, all results are shown in the deformed configuration.

\section{Interaction of phase transformations and dislocations in nanograins under plas- tic shear}

Since simple shear represents the main mechanism of plastic flow at several scales, it is of great importance to study the interaction between PT and plasticity for this type of loading. Simple shear or close to simple shear conditions can be realized under torsion, high-pressure torsion (Perez-Prado and Zhilyaev (2009); Valiev et al. (2000); Zhilyaev and Longdon (2000); Valiev et al. (2000)), friction, cutting, scratching, and polishing (Gogotsi and Domnich (2004); Patten et al. (2005)), and ball milling (Boldyrev (2006); Delogu (2012)). Instability in the form of shear band localization may occur at various scales, from a nanoscale to a kilometer scale in geophysical applications. Plastic strain-induced PTs under such conditions may lead to the desired phases (Perez-Prado and Zhilyaev (2009); Valiev et al. (2000); Zhilyaev and Longdon (2000); Valiev et al. (2000); Boldyrev (2006); Delogu (2012)), to an earthquake (Green and Burnley (1989); Kirby (1987)), or to a ductile regime of machining otherwise brittle semiconductors (e.g., Si and Ge) and ceramics (e.g., SiC), see Gogotsi and Domnich (2004); Patten et al. (2005). PTs under superposition of high pressure and large plastic shear in rotational Bridgman anvils (Bridgman (1937)) and rotational diamond anvil cell (Levitas et al. (2012); Blank et al. (2007); Ji et al. (2012); Levitas (2004a)) lead to new phases or known phases under much lower pressure than under hydrostatic conditions.

At the microscale, PTs under simple shear with normal stress or prescribed normal and shear stresses, or in a shear band have been treated analytically in Levitas (1995, 1997, 1998, 2002); Levitas et al. (1998a,b); Levitas (2000), including finite-strain formulation. Microscale thermodynamic (Levitas $(1997,1998)$ ) and kinetic (Levitas (2000)) theories for PTs in elastoplastic materials with sharp interfaces have been utilized. Homogeneous evolving stress and 
strain states were considered. Under prescribed shear stresses, PTs induced transformationinduced plasticity (Levitas $(1998,2002)$ ) or (with similar formalism) reaction-induced plasticity (Levitas et al. (1998a,b); Levitas (2000)).

In Levitas (2004a,b), the following mechanism of significant reduction of PT pressure due to plastic shear was suggested: shear stresses produce dislocation pile-up with strong stress concentration, which leads to barrierless $\mathrm{M}$ nucleation near its tip. This problem was solved analytically in Levitas (2004a,b). A phase field solution for this problem under prescribed shear strain and normal stresses have been found and analyzed in Levitas and Javanbakht (2014a). Here, we solve the problem on simultaneous dislocation and $\mathrm{M}$ evolution under prescribed simple shear in a nanosize bicrystal and analyze results in detail. For better understanding of underlying physics, it is desirable to separate effects of pressure and plastic shear and to find both nanoscale detail and possibility of macroscale characterization of such PTs. Nanograins are chosen because large plastic deformations lead to nanograin structure and because we resolve a nm sized interface width and dislocation core, the consideration of a large-grain sample is computationally expensive.

\subsection{Problem formulation}

Sample. A square sample is considered with the size of $30 \times 30$, which includes the following regions (Fig. 2):

(a) A rectangle with the size of $15 \times 20$ located on the left side of the sample at the middle of which a horizontal dislocation system (defined by the order parameter $\xi_{3}$ ) is considered. In this region (left grain), the mechanical and dislocation problems are solved. The slip system is chosen in parallel to the shear direction to model the optimally oriented grain.

(b) Two rectangular regions with the size of $30 \times 5$ located at the top and the bottom, and a rectangle with the size of $5 \times 20$ located at the right side of the sample where the mechanical problem is solved only. These regions model elastic accommodation of the grains that surround two grains with plasticity. We chose relatively small width of the right accommodating region near the free surface in order to mimic that additional stress relaxation in it may occur through PT and plasticity.

(c) A rectangular region with the size of $10 \times 20$ located inside the sample (right grain), 
surrounded by regions $a$ and $b$, where two dislocation systems (defined by the order parameter $\xi_{1}$ and $\left.\xi_{2}\right)$ inclined at $\pm 30^{\circ}$ are included. In this region, equations for both the mechanical and PT problems, or for the mechanical, PT, and dislocation problems are solved.

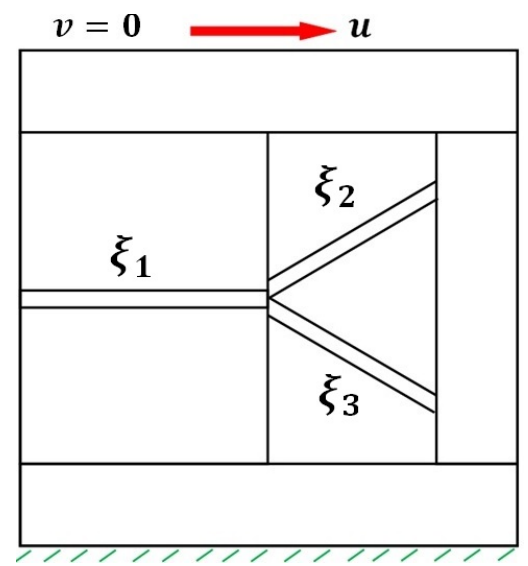

Figure 2: (color online) Schematics of the sample under simple shear. A horizontal slip system is included in the middle of the left grain. Two slip systems inclined at $\pm 30^{\circ}$ are considered when plasticity is included in the right grain.

Boundary conditions. For the mechanical problem, the lateral sides are stress-free. We solved to some extend similar problem in Levitas and Javanbakht (2014a) under periodic boundary conditions at the vertical sides and have an idea on what to expect from these conditions for the current problem. That is why we chose here elastic region on the right side that mimics elastic accommodation and (due to relatively small width) accommodation due to PT and plasticity in a neighboring grain. We did not include similar region on the left side, because we found that it practically did not affect the PT. Also, we wanted to demonstrate that we can solve problems for nonperiodic boundary conditions, which cannot be done in the majority works in PFA due to utilization of spectral methods of a problem solution. For macroscopically simple shear the lower side is fixed; the upper side is fixed in the $y$ direction and is subjected to the following homogeneous horizontal displacement $u: u=1.4+t$ from $t=0$ to 2.8 , then $u=4.2$ from $t=2.8$ to 11.2 . The horizontal displacement will be mentioned below in terms of prescribed macroscopic shear $\gamma=u / h$, with the height of grains $h=20$. Boundary conditions for order parameters are given in Eq.(21).

Initial conditions. As the first step in preparation of the initial conditions for all problems, GL equations for dislocations only coupled to mechanics are solved in the left grain only without considering PT. Initial condition is $\xi_{3}=0.01$ within a slip band. Under the prescribed 
shear until $t=5.6,3$ dislocations are generated inside the dislocation band in the left grain. Fig. 3 presents the evolution of dislocations in the left grain. A dislocation sign shows the slip plane and the extra half plane of atoms producing a dislocation, as usual. As can be seen, the first positive dislocation nucleates at the grain boundary creating a step at the grain boundary. A negative dislocation, which nucleates near the grain boundary propagates to the left side. The next dislocation pairs nucleate near the grain boundary as well. The second and third negative dislocations propagate to the left and create a pile-up at the left side of the left grain. The second and third positive dislocations move to the right and are densely piled up at the grain boundary. This pile-up creates a strong concentration of the stress tensor near its tip. This leads to an increase in the local thermodynamic driving force for PTs in the region near the dislocations tip in the right grain, which causes $M$ nucleation. We found that 3 is the smallest number of dislocations in the left grain required to create a stress concentration large enough to cause $\mathrm{M}$ nucleation in the right grain. In fact, the initial condition $\eta=0.01$ in the right grain evolves to zero for less than 3 dislocations in the left grain. Thus, such a solution with 3 dislocation couples in the left grain is considered as an initial condition for two different problems on PT evolution in the right grain. We also accept $\mathrm{M}$ perturbation $\eta=0.01$ everywhere and $\xi_{i}=0.01$ within each of slip systems, both in the right grain.

Types of problems. Two types of problem will be considered to determine the competition between PT and plasticity in the right grain:

1. Plasticity is not included in the right grain.

2. Dislocation activity along two slip systems inclined at $\pm 30^{\circ}$ in the right grain, which competes with the PT, is included.

Problems 1 and 2 are solved for 4 different cases of transformation strains and temperatures (i.e., thermal driving force), which result in different $M$ nuclei and regions with different $M$ concentrations. For each case, $\mathrm{M}$ for problem 1 (no plasticity in the right grain) is compared with that of problem 2 (plasticity included), and the effects of dislocations and their interactions with PT are elucidated. As will be seen later, some of these examples show a significant effect of plasticity on PT, while others do not. The transformation strains and temperature, which define mechanical and thermal driving forces for PT, for these four cases are chosen as follows:

case 1: $\quad \varepsilon_{t}^{x}=-0.1, \varepsilon_{t}^{y}=0, \varepsilon_{t}^{x y}=0.1$, and $T=600 K$; 


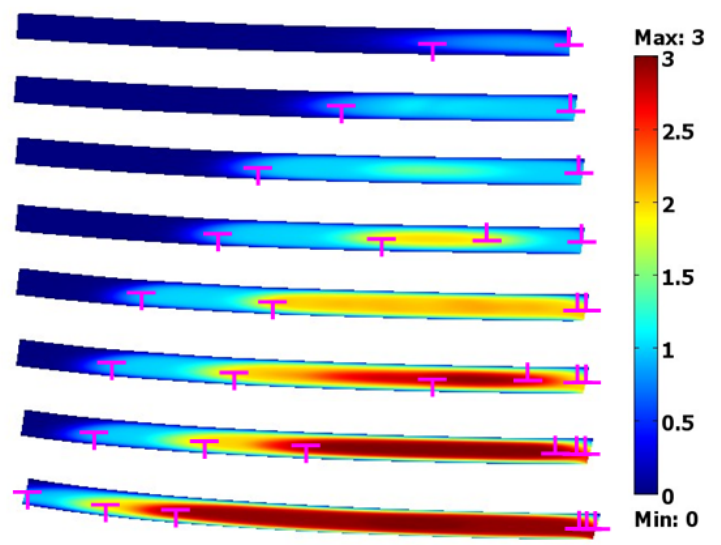

Figure 3: (color online) The evolution of dislocations in the left grain under prescribed shear displacement.

case 2: $\quad \varepsilon_{t}^{x}=-0.1, \varepsilon_{t}^{y}=-0.04, \varepsilon_{t}^{x y}=0.1$, and $T=800 K$

case 3: $\quad \varepsilon_{t}^{x}=-0.1, \varepsilon_{t}^{y}=-0.05, \varepsilon_{t}^{x y}=0.1$, and $T=1000 K$;

case 4: $\quad \varepsilon_{t}^{x}=-0.1, \varepsilon_{t}^{y}=-0.09, \varepsilon_{t}^{x y}=0.1$, and $T=1000 K$.

Material parameters. The following material parameters for cubic to tetragonal PT in $\mathrm{NiAl}$ have been calibrated in Levitas and Preston (2002b); Levitas and Javanbakht (2010, 2011a,b): $\beta_{\eta}^{A}=\beta_{\eta}^{M}=2.59 \cdot 10^{-10} N, L_{\eta}^{A}=L_{\eta}^{M}=2600(\mathrm{~Pa} \cdot s)^{-1}, A_{0}=4.4 \mathrm{MPaK}^{-1}, \Delta s=-A_{0} / 3, a=3$, $\theta_{e}=215 K$, and $\theta_{c}=-183 K$. They correspond to the equilibrium interface width of $0.73 \mathrm{~nm}$ and energy $0.159 \mathrm{~J} / \mathrm{m}^{2}$.

Material parameters for dislocations are taken from Levitas and Javanbakht $(2012,2013)$ : $L_{\alpha}^{A}=L_{\alpha}^{M}=10^{4}(\mathrm{~Pa} \cdot s)^{-1}, \beta_{\xi}^{A}=\beta_{\xi}^{M}=1.09 \cdot 10^{-10} \mathrm{~N}, A_{\alpha}^{A}=0.894 G P a$ for $\mathrm{A}, A_{\alpha}^{M}=2.68 G P a$ for $\mathrm{M}, Z=0.05, H^{\alpha}=0.7 n m,\left|\boldsymbol{b}_{\alpha}\right|=0.35 \mathrm{~nm}, \gamma_{\alpha}=0.25$. Here, we consider that the critical shear stress for barrierless nucleation of dislocation in $M$ is 3 times larger than that for $A$.

\subsection{Numerical solutions}

For $\gamma=0.21$ (at $t=5.6$ ), the solution corresponds to three dislocation couples in the left grain, resulting in average shear stresses $\bar{\tau}=6.37$ and $\bar{\tau}=8.21$ in the left and right grains, respectively, and practically zero pressure $(p=-1 / 3 \boldsymbol{\sigma}: \boldsymbol{I})$ for both grains. Such a solution is considered as an initial condition for the following problems. At this time instant we start the solution of the Ginzburg-Landau equations in the right grain either just for PT (corresponding to problem 1) or for PT and two inclined dislocation systems (corresponding to problem 2). After a solution is found, the concentration of the $M$ is defined with respect to the grain area 
in the undeformed state:

$$
c=\int \eta d V_{0} / V_{0}
$$

where, both here and below, averaging over the volume is equivalent to averaging over the area. An alternative definition is $c=\int \varphi(a, \eta) d V_{0} / V_{0}$, because all properties depend linearly on $\varphi$ just as on concentration. However, for the untransformed region $\eta=\varphi=0$, for complete $\mathrm{M} \eta=\varphi=1$; thus, the difference is only at interfaces and in the incomplete $\mathrm{M}$ areas, and for most of our simulations it can be neglected.

\subsubsection{Problem 1 (no plasticity in the transformed grain).}

Microstructure. $\mathrm{M}$ nucleates barrierlessly due to a large stress concentration at the tip of the dislocation pile-up and grows both in longitudinal and lateral directions until interfaces reach a thermodynamic equilibrium due to the reduction of the effect of stress concentration and generation of internal stresses due to transformation strain (Fig. 4). Note that during the evolution of $\mathrm{M}$ in the right grain, dislocations in the left grain also evolve and the number of dislocations increase. It is found that even though the $\mathrm{M}$ evolution, and consequently the degrees of stress relaxation are different for all the cases, the number of dislocations in the left grain increases to 6 . Thus, the dislocation activity in the left grain is mainly determined by the applied shear and is independent of the $\mathrm{M}$ nucleation and evolution.
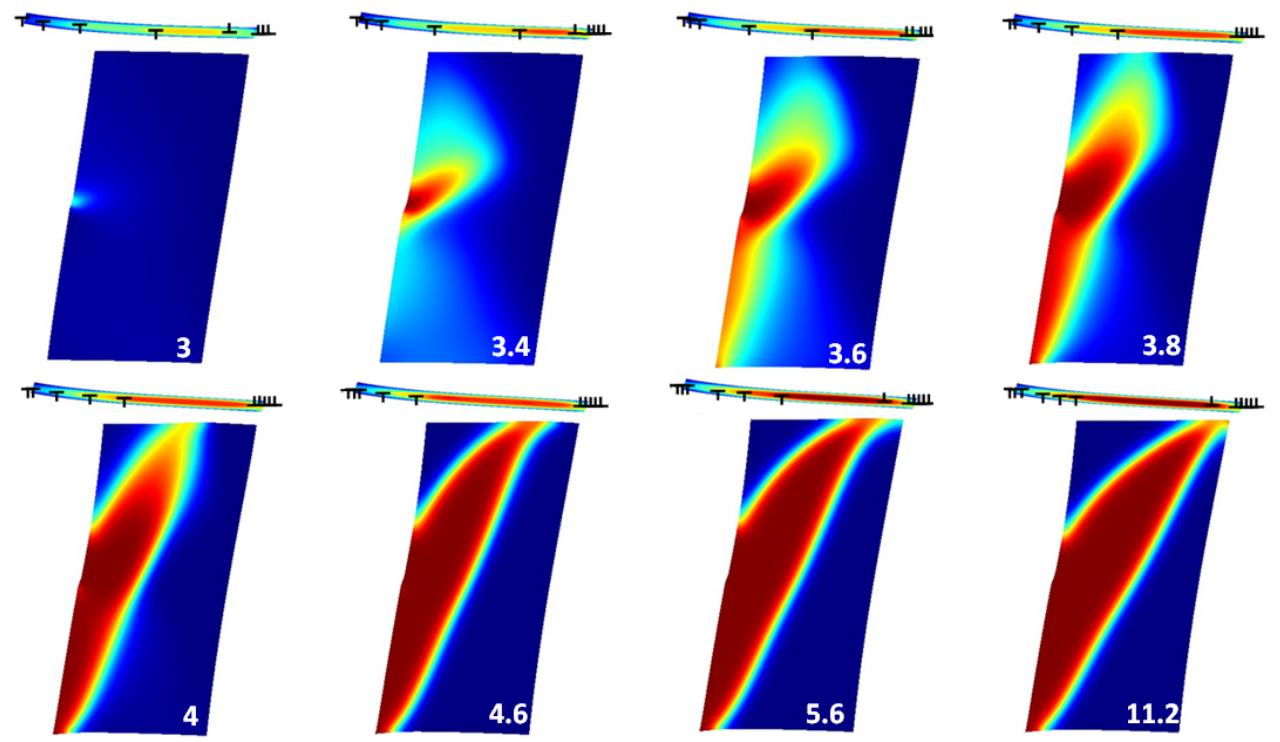

Figure 4: (color online) Evolution of martensitic nanostructure in the right grain (without plasticity) and corresponding dislocation system of the left grain for case 2 . Time 0 in the figures corresponds to the instant when PT is included. 
The stationary $\mathrm{M}$ nanostructures for cases 1 to 4 are presented in Fig. 5 (left column in each box), with concentrations of $\mathrm{M} c_{1}=0.79, c_{2}=0.47, c_{3}=0.26$, and $c_{4}=0.12$, respectively (see Table 1). Increasing temperature and $\left|\varepsilon_{t}^{y}\right|$ under constrained conditions suppresses PT. Also, corresponding stationary dislocation structures in the left grain are included for each case.
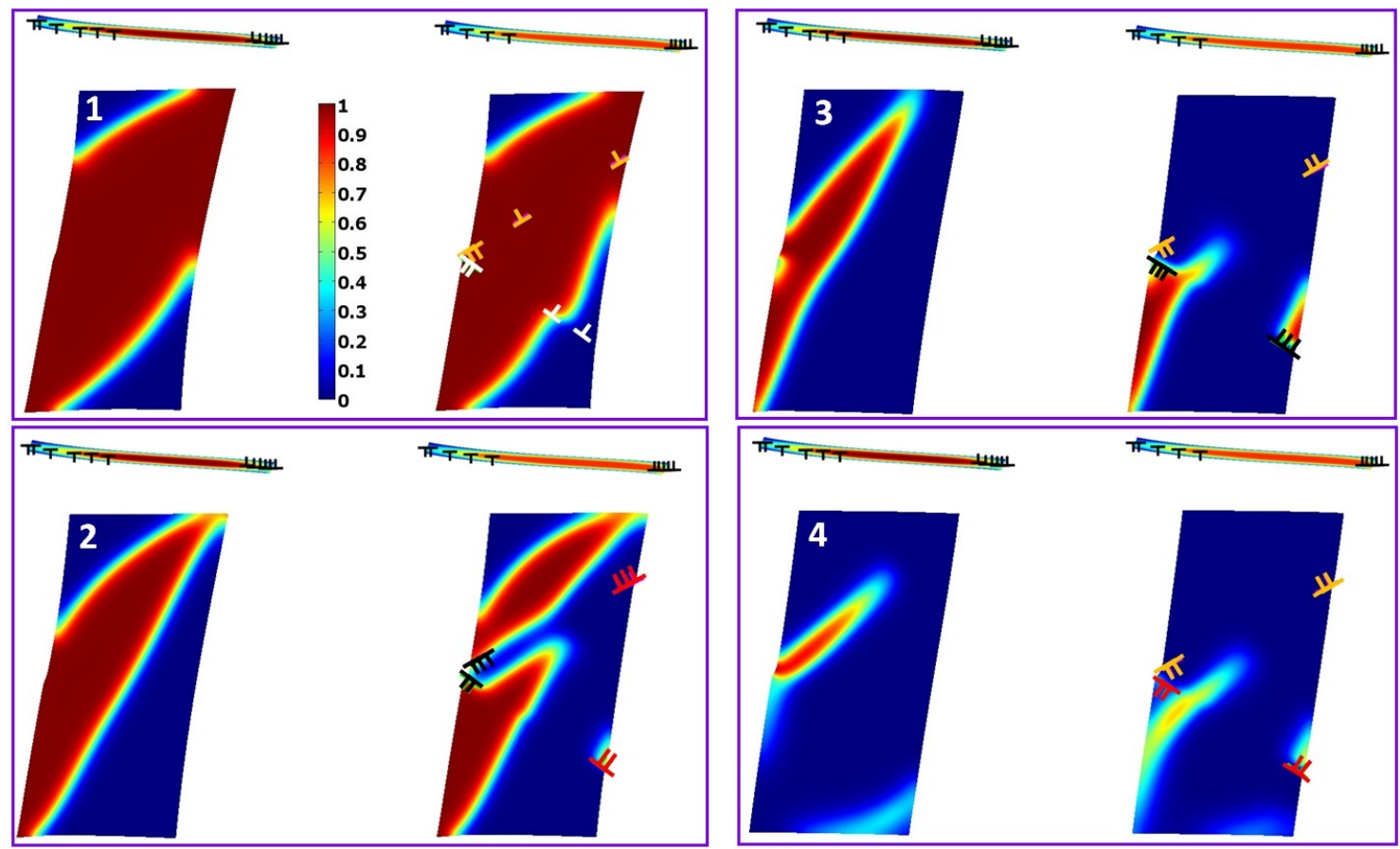

Figure 5: (color online) Stationary martensitic and dislocational microstructures in the right grain for cases 1 to 4 . The left and right columns for each case correspond to PT without and with plasticity in the right grain, respectively. Dislocation structure in the left grain are shown above the right grain. The vertical legend is for the PT order parameter $\eta$ and will be the same for all figures in the paper.

For cases 3 and 4, the morphology of the $\mathrm{M}$ nucleus within a nanoscale sample is qualitatively in agreement with the analytical model for a $\mathrm{M}$ nucleus induced by a dislocation pile-up in an infinite media (Levitas (2004b)). For cases 1 and 2, longitudinal growth is arrested by the grain boundary and thickening occurs until thermodynamically equilibrium width is achieved.

Pressure evolution. Note that pressure averaged over the grain $\bar{p}$ is tensile (negative) and its magnitude increases during $\mathrm{PT}$ in both grains. Also, the shear stress $\bar{\tau}$ averaged over the grain is relaxed due to the plastic and transformational shear deformation in both grains. Since in experiment PT pressure is measured after the PT and at the place where the PT occurred, we will report $\bar{p}$ and $\bar{\tau}$ after PT in the transformed grain as well. For cases 1 to 4 , the averaged tensile pressure $\bar{p}$ evolves to the following values for the left and right grains, respectively: for 
case $1, \bar{p}_{l}=-0.41$ and $\bar{p}_{r}=-1.11 ;$ for case $2, \bar{p}_{l}=-0.25$ and $\bar{p}_{r}=-2.13$; for case $3, \bar{p}_{l}=-0.14$ and $\bar{p}_{r}=-1.41$; and for case $4 \bar{p}_{l}=-0.04$ and $\bar{p}_{r}=-1$. Fig. 6 illustrates the averaged pressure $\bar{p}$ for both grains vs. the $\mathrm{M}$ concentration in the right grain. The averaged pressure in the left grain is linearly but weakly dependent on the concentration, while in the right grain, it linearly decreases with increasing concentration to $c=0.47$, and then increases with increasing concentration to $c=0.79$. Nonmonotonous dependence of the averaged pressure for increasing $\left|\varepsilon_{t}^{y}\right|$ and $c$ demonstrates a complex character of stress redistribution within and between grains.

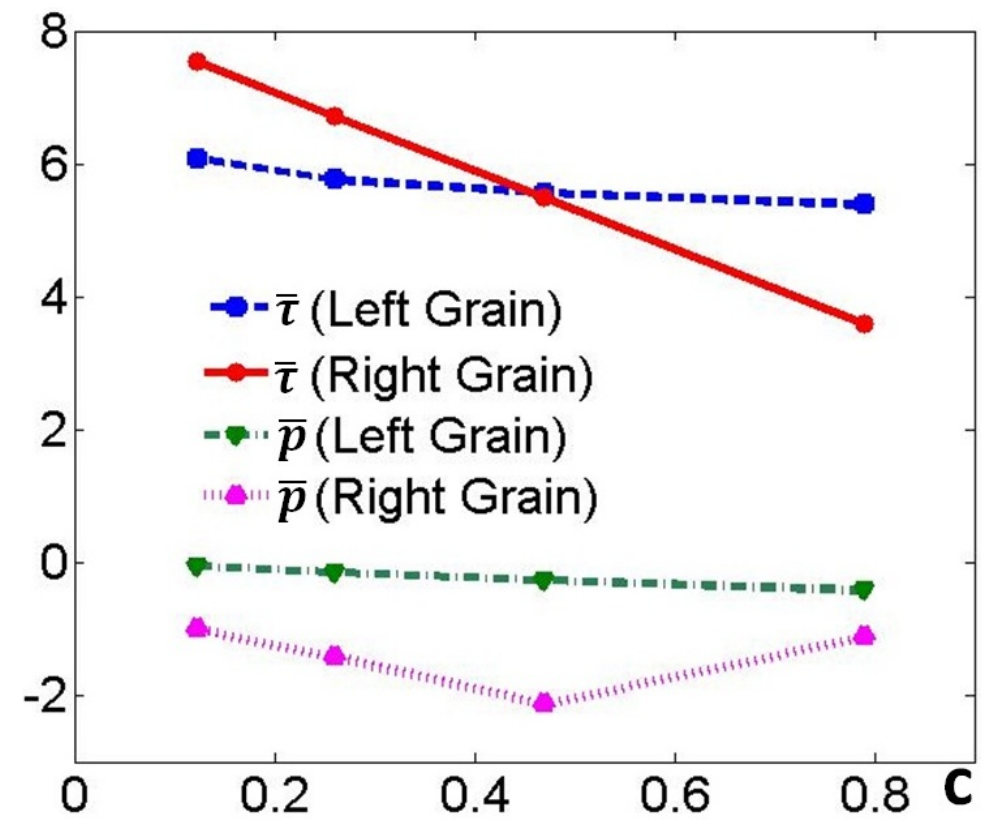

Figure 6: (color online) Averaged pressure and shear stress over left and right grains vs. M concentration for problem 1 without plasticity in the transformed grain.

The evolution of pressure toward the stationary solution in the right grain for case 2 is presented in the first row of Fig. 7. Initially, there is a growing negative pressure concentrator near the tip of the dislocation pile-up (due to the increasing number of dislocations) and large negative and positive pressure concentrators in the right upper and lower corners of the grain. Pressure redistribution occurs due to transformation strain in the growing $M$ region under constrained conditions. Also, it is found that the pressure is negative (tensile) in a large area inside the grain, which suppresses PT; despite this, $\mathrm{M}$ is mostly located within this region due to large shear stresses.

Shear stress evolution. For cases 1 to 4, the averaged shear $\bar{\tau}$ is also reduced to the following values for the left and right grains, respectively: for case $1, \bar{\tau}_{l}=5.4$ and $\bar{\tau}_{r}=3.6$; for case 2 , 

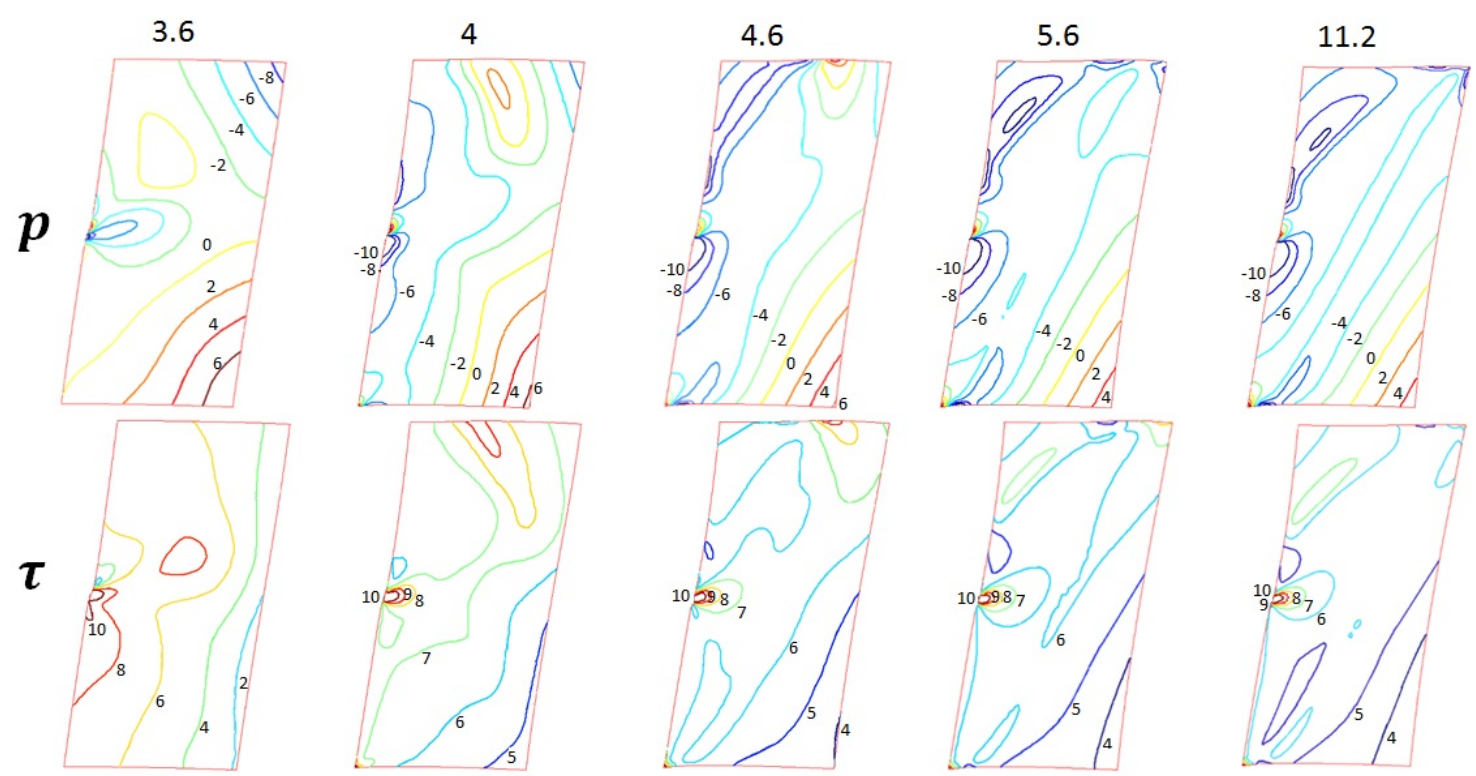

Figure 7: (color online) Pressure and shear stress contour lines evolution in the right grain without plasticity for case 2 .

$\bar{\tau}_{l}=5.57$ and $\bar{\tau}_{r}=5.5 ;$ for case $3, \bar{\tau}_{l}=5.77$ and $\bar{\tau}_{r}=6.72 ;$ and for case $4, \bar{\tau}_{l}=6.1$ and $\bar{\tau}_{r}=7.54$. For a case with a small and negligible $\mathrm{M}$ concentration (cases 3 and 4), relaxation of shear stress is relatively small and mostly in the left grain due to dislocation activity. That is why shear stresses are the highest for these cases and are larger in the right grain. With increasing $M$ concentration (cases 1 and 2), shear stress relaxation is more pronounced especially in the right grain. That is why shear stress reduces in both grains but at a higher rate in the right grain than in the left.

The averaged shear stress $\bar{\tau}$ (Fig. 6) linearly decreases with the concentration of $\mathrm{M}$ in both grains but at a higher rate in the right grain than in the left. Initially, $\bar{\tau}$ in the right grain is larger but above some $\mathrm{M}$ concentration is getting smaller than in the left grain. The evolution of shear stress in the right grain is presented in Fig. 7 for case 2. During the evolution to the stationary solution, the shear stress relaxes in regions away from the tip of dislocations but it grows near the tip of dislocations due to an increasing number of dislocations.

\subsubsection{Problem 2 (with plasticity in the transformed grain).}

Microstructure. For problem 2, PT and dislocation evolution along two inclined slip systems are allowed in the right grain at $t=5.6$. Due to the stress concentration, $\mathrm{M}$ and dislocations along both slip systems nucleate in the right grain near the tip of the dislocation pile-up in the left grain. Due to the stress relaxation via the nucleation of dislocations, $\mathrm{M}$ nucleation and 
growth, and consequently the stationary nanostructure and concentration are much different than that for problem 1 without dislocations in the right grain. The stationary M nanostructures for cases 1 to 4 are represented in Fig. 5 (right column in each box), with concentrations $c_{1}=0.76, c_{2}=0.39, c_{3}=0.1$, and $c_{4}=0.06$, respectively (Table 1 ). Also, the corresponding stationary dislocations in the left and right grains for each case are included. For all the cases, the number of dislocations in the left grain increases from 3 to 5 , instead of to 6 for problem 1. This is caused by the larger stress relaxation due to generation of dislocations along two inclined slip systems in the right grain, which suppresses dislocation generation in the left grain. For cases 1 to 4 , both $\pm 30^{\circ}$ slip systems contain two stationary dislocations, except for case 2 with three dislocations inclined under angle $+30^{\circ}$, and case 3 with 3 dislocations inclined under angle $-30^{\circ}$. The stationary $\mathrm{M}$ nanostructure for case 1 is very close to that for problem 1, however the stationary solutions for case 2 to 4 are different from those of problem 1 , and the concentrations of $\mathrm{M}$ are smaller. This is because of the larger stress relaxation due to the nucleation and evolution of dislocations, which suppresses $\mathrm{M}$ nucleation and growth. Thus, for case 2, an $\mathrm{A}$ band divides a $\mathrm{M}$ nucleus into two smaller nuclei, leading to morphological transition. For case 3, a smaller nucleus appears near the left grain boundary but the second nucleus appears near the pile-up of three dislocations near the right boundary. For case 4, M embryos are not even complete, i.e., $\eta$ did not reach unity.

The evolution of the $\mathrm{M}$ nanostructure in the right grain and corresponding dislocation structure in the left and right grains are presented for case 2 in Fig. 8. First, two dislocation pairs in each of the two slip systems nucleate and produce a pile-up at the grain boundaries. Then two $\mathrm{M}$ nuclei appear above and below the dislocation band inclined under $+30^{\circ}$. When their evolution is almost completed, the third dislocation pair nucleates in the slip system inclined under $+30^{\circ}$, which also piles up near the grain boundaries.

Pressure evolution. For cases 1 to 4, the averaged tensile pressure $\bar{p}$ grows to the following values for the left and right grains, respectively: for case $1, \bar{p}_{l}=-0.38$ and $\bar{p}_{r}=-1.18$; for case $2, \bar{p}_{l}=-0.23$ and $\bar{p}_{r}=-1.72$; for case $3, \bar{p}_{l}=-0.12$ and $\bar{p}_{r}=-0.8$; and for case 4 $\bar{p}_{l}=-0.04$ and $\bar{p}_{r}=-0.49$. Fig. 9 illustrates the averaged pressure $\bar{p}$ for both grains vs. the $\mathrm{M}$ concentration in the right grain. Similar to problem 1, the averaged pressure in the left grain is linearly but weakly dependent on the concentration, while in the right grain, it 

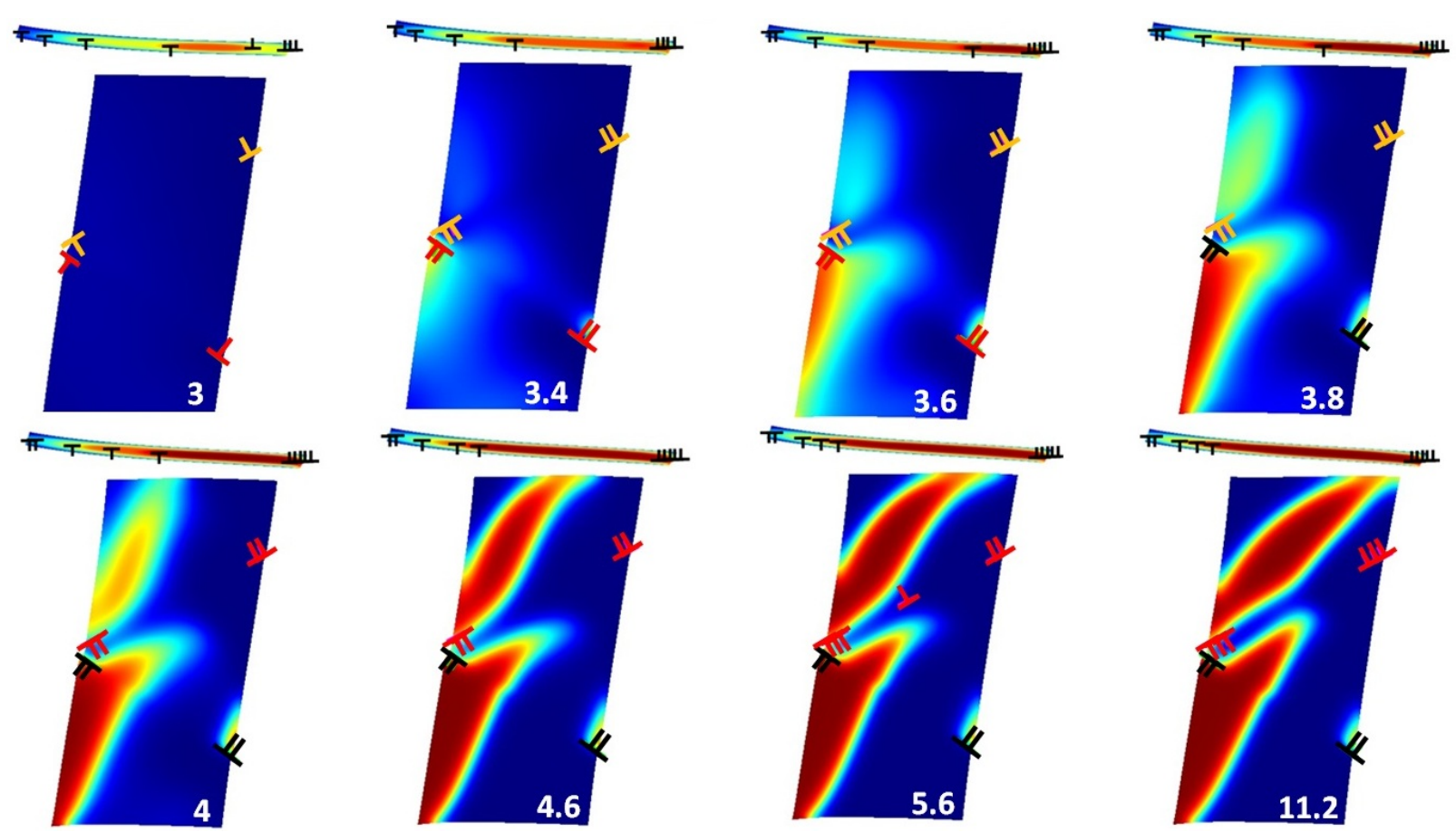

Figure 8: (color online) The evolution of martensite and dislocations in the right grain for case 2. The dislocation band in the left grain is plotted on the top of each right grain.

linearly decreases with increasing concentration to $c=0.47$, and then increases with increasing concentration to $c=0.79$. Also, generally the magnitude of the tensile pressure in each grain for problem 2 is smaller than that of problem 1 . The reason is in a smaller concentration of $\mathrm{M}$ and consequently smaller volume reduction in comparison with problem 1.

The evolution of pressure in the right grain is presented in the first row of Fig. 10. There are pressure concentrations near the tip of dislocations at the left and right boundaries, which slightly relax during evolution.

Shear stress evolution. Also, for cases 1 to 4 , the averaged shear $\bar{\tau}$ is reduced to the following values for the left and right grains, respectively: for case $1, \bar{\tau}_{l}=5.38$ and $\bar{\tau}_{r}=3.39$; for case $2, \bar{\tau}_{l}=5.55$ and $\bar{\tau}_{r}=5.38$; for case $3, \bar{\tau}_{l}=5.85$ and $\bar{\tau}_{r}=7.06$; and for case 4 , $\bar{\tau}_{l}=6$ and $\bar{\tau}_{r}=7.39$. Similar to problem 1, the averaged shear stress $\bar{\tau}$ linearly decreases with increasing concentration of $\mathrm{M}$, weakly in the left grain and stronger in the right grain (Fig. 9). The evolution of shear stress in the right grain is presented in Fig. 10. There are huge concentrations of shear stress near the tips of dislocation pile-ups at the left and right boundaries. Also, some contour lines of shear stress are oriented along slip planes. The average shear stress $\bar{\tau}$ vs. the average shear strain $\bar{\varepsilon}^{x y}$ in the right grain is also plotted for the cases 1 


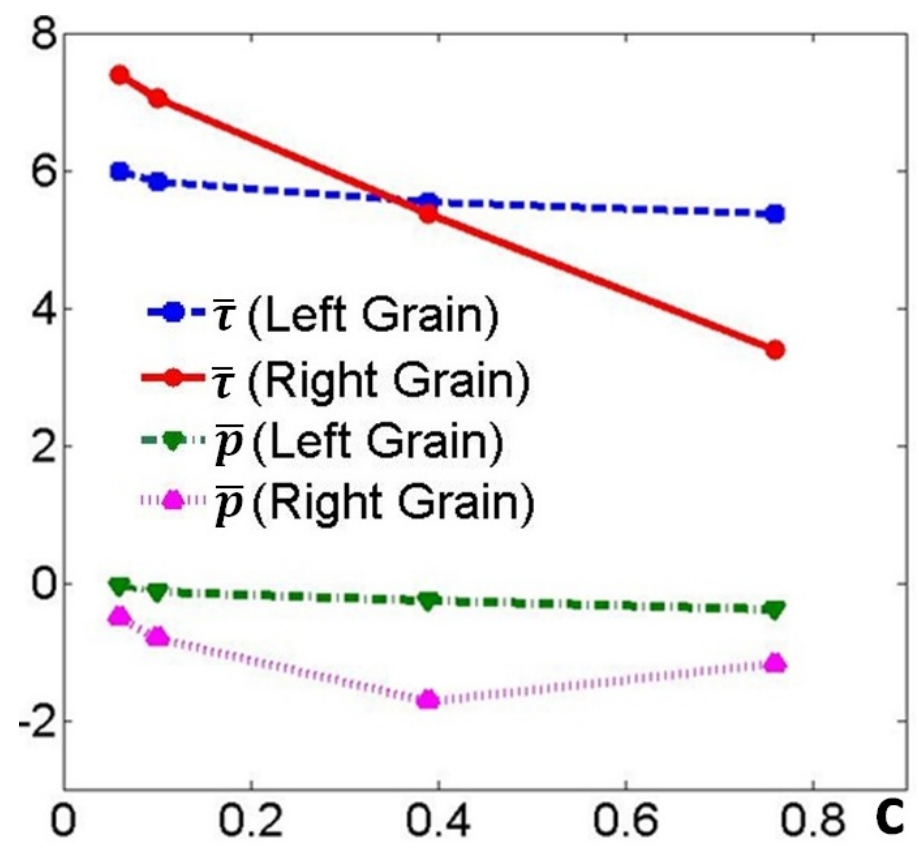

Figure 9: (color online) Averaged pressure and shear stress over left and right grains vs. $M$ concentration for problem 2 with plasticity in the transformed grain.
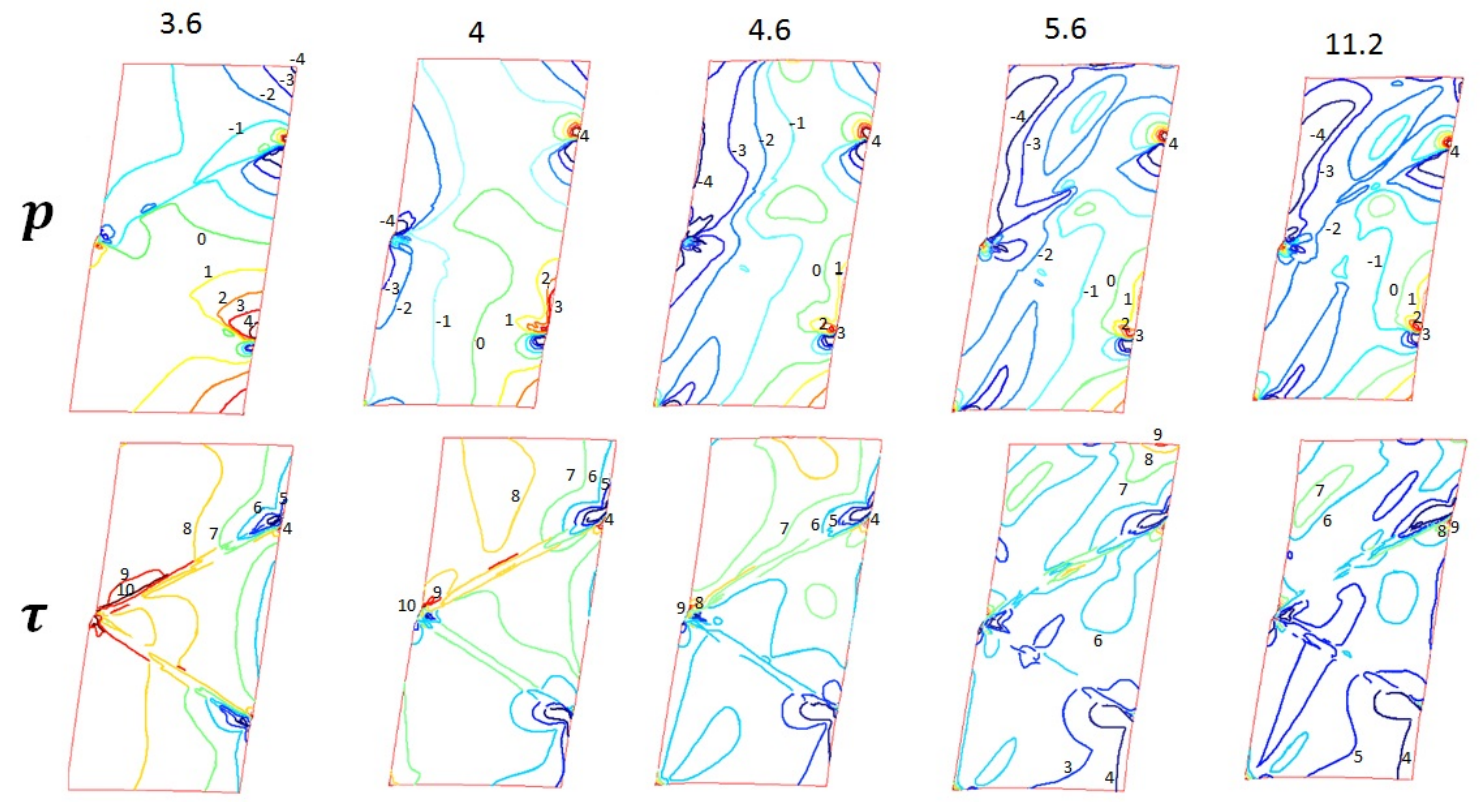

Figure 10: (color online) Pressure and shear stress contour lines evolution in the right grain with plasticity for case 2.

and 2 in Figs. 11a and b, respectively. Results without and with plasticity are almost the same, implying that phase transformations and dislocations play the same part in stress relaxation.

\subsubsection{Transformation work.}

Here, we will try to interpret stationary geometry of the $\mathrm{M}$ region and $\mathrm{M}$ concentration in 

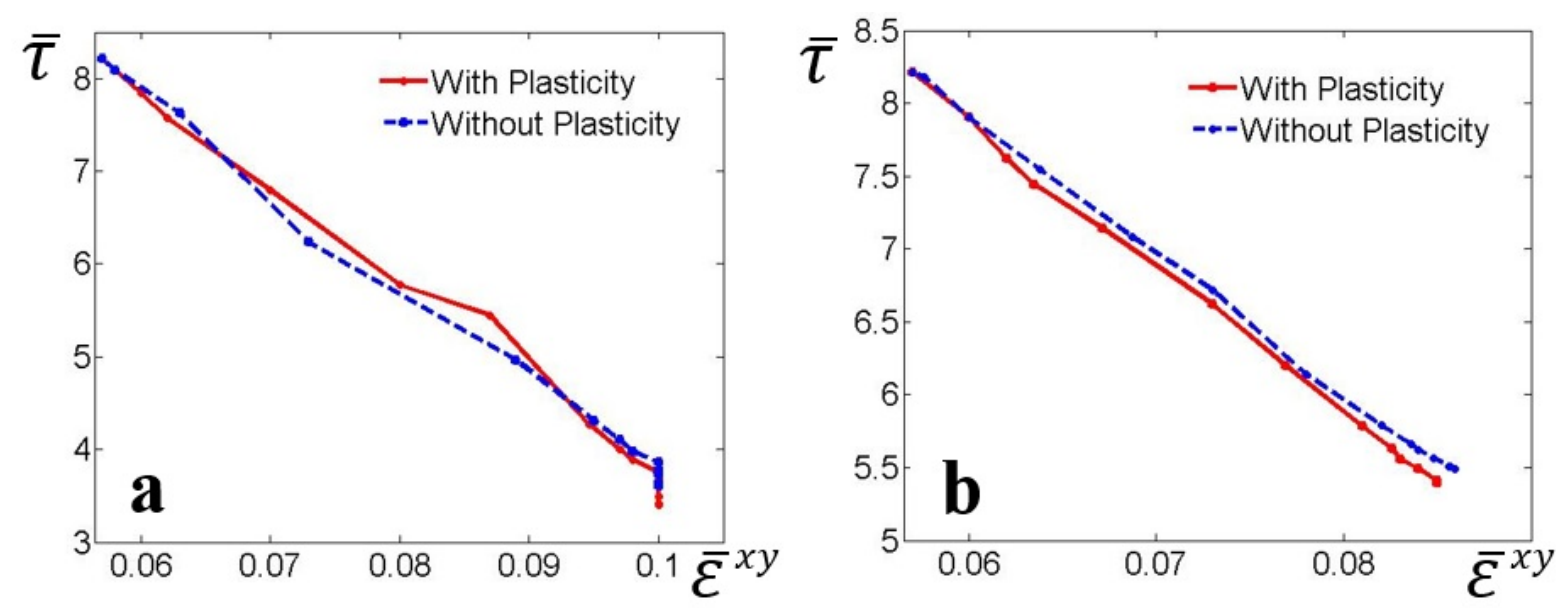

Figure 11: (color online) The average shear stress $\bar{\tau}$ vs. the average shear strain $\overline{\boldsymbol{\varepsilon}}^{x y}$ in the right grain for case 1 (a) and case 2 (b).

terms of approximate thermodynamic equilibrium conditions across a stationary interface and estimates for transformation work. For simplicity, we will neglect interface energy and calculate transformation work such as in a geometrically linear approximation. Then the local phase equilibrium condition for each point of an interface in the current configuration is (Levitas $(1997,1998,2000,2002))$

$$
\boldsymbol{\sigma}: \varepsilon_{t}=\Delta G^{\theta}(\theta)=\left(A_{0} / 3\right)\left(\theta-\theta_{e}\right) .
$$

An important point of Eq. (23) is that plastic strain does not contribute to it, in contrast to the approach based on utilizing the Eshelby driving force (Cherkaoui et al (1998); Cherkaoui and Berveiller (2000); Fischer and Reisner (1998); Fischer et al. (2000)). This aspect was analyzed in detail in Levitas (2002).

For temperatures under study, values $\Delta G^{\theta}(\theta)$ are presented in Table 1. Using local distributions of stress and transformation strain tensors in the right grain, one can calculate the field of the transformation work $\boldsymbol{\sigma}: \boldsymbol{\varepsilon}_{t}$. For each temperature, Eq. (23) determines the contour line along which simplified phase equilibrium condition is satisfied.

The stationary $\mathrm{M}$ morphology and contour lines of the equilibrium values of PT work are plotted in the right grain for problems with and without plasticity for cases 1 and 2 (Fig. 12). For case 1 , interface positions perfectly coincide with the lines corresponding to the equilibrium PT work, both with and without plasticity (Fig. 12 a and b). Note that in these cases interfaces are away from the strong stress concentrators. For case 2, interface positions coincide with the 
lines corresponding to the equilibrium PT work in the major part of the interfaces, both with and without plasticity (Fig. $12 \mathrm{c}$ and d). However, there is a deviation between these lines in the middle of the grain for both cases and near the tip of the dislocation pile-up. In the middle of the grain, matching the interface to the equilibrium PT work line would require a significant increase in the interface area (length) and energy, as well as additional internal stresses due to the curved interface. The deviation between these lines near the tip of the dislocation pile-up is due to very high stress heterogeneity.

Note that there are regions where PT work exceeds the equilibrium value but $\mathrm{M}$ does not appear. This happens for several reasons. First, stresses did not reach the level required for barrierless nucleation. Second, even if they reached it, the appearance of the nucleus produces significant interface and elastic energy, so that nucleation is energetically unfavorable. And third, actually introducing $\mathrm{M}$ in these regions may redistribute stresses and reduce PT work below its equilibrium values.
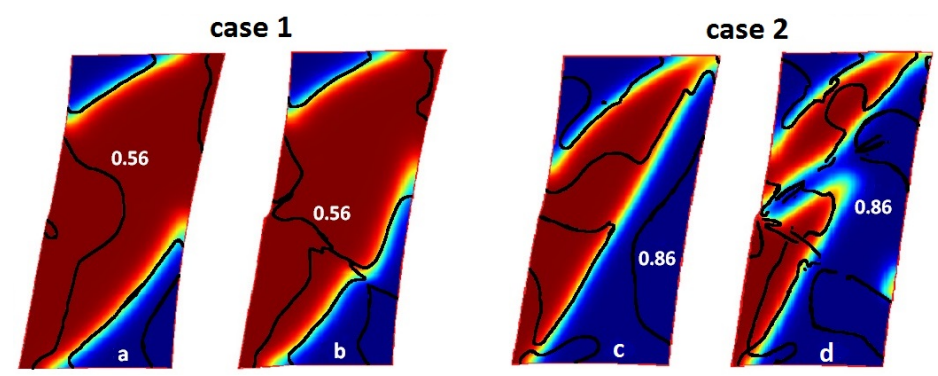

Figure 12: (color online) Stationary phase state and contour line of the equilibrium transformation work for case 1 without (a) and with plasticity (b), and for case 2 without (c) and with plasticity (d).

To elucidate this further in terms of the fields averaged of the transformed and total grain area, let us define the averaged stationary PT work

$$
W_{t}=\frac{1}{V_{0}} \int \boldsymbol{\sigma}: \varepsilon_{t}(\eta) d V_{0} \simeq \frac{1}{V_{0}} \int \boldsymbol{\sigma}: \varepsilon_{t} d V_{M} \simeq \frac{c}{V_{M}} \int \boldsymbol{\sigma}: \varepsilon_{t} d V_{M}=\left\langle\boldsymbol{\sigma}: \varepsilon_{t}>_{M} c=<\boldsymbol{\sigma}>_{M}: \varepsilon_{t} c\right.
$$

where $<\ldots>_{M}=\frac{1}{V_{M}} \int \ldots d V_{M}$ is a parameter averaged over the $\mathrm{M}$ area. In the above approximations we assume that interface is thin in comparison with the bulk $\mathrm{M}$ region and utilize the fact that $\varepsilon_{t}(\eta)$ is nonzero and homogeneous in the $\mathrm{M}$ region. We also introduce the averaged PT work for a fully transformed grain with the same stress distribution

$$
W_{t}^{*}=\frac{1}{V_{0}} \int \boldsymbol{\sigma}: \varepsilon_{t} d V_{0}=<\boldsymbol{\sigma}: \varepsilon_{t}>_{0}=<\boldsymbol{\sigma}>_{0}: \varepsilon_{t}
$$

in terms of averaged values over the entire grain $<\ldots>_{0}=\frac{1}{V_{0}} \int \ldots d V_{0}$. The counterpart of the 
local thermodynamic equilibrium condition (23) in the averaged description are

$$
\begin{array}{r}
<\boldsymbol{\sigma}>_{M}: \varepsilon_{t}=\Delta^{\theta} G(\theta)=\left(A_{0} / 3\right)\left(\theta-\theta_{e}\right) \quad \text { or } \\
<\boldsymbol{\sigma}>_{0}: \varepsilon_{t}=\Delta^{\theta} G(\theta)=\left(A_{0} / 3\right)\left(\theta-\theta_{e}\right),
\end{array}
$$

and due to the approximate character of these conditions there is not a priory way to prefer one over another. Results of simulations of the parameters $\left\langle\boldsymbol{\sigma}>_{M}: \varepsilon_{t},<\boldsymbol{\sigma}>_{0}: \varepsilon_{t}, W_{t}\right.$, as well as normalized parameters $W_{t} / \Delta^{\theta} G(\theta)$ and $W_{t} / W_{t}^{*}$ for the problems without and with plasticity for all cases are presented in Table 1 and Fig. 13. They demonstrate quite good fulfilment of averaged equilibrium criteria (26) and (27), which also means the equality of two averaged stress tensors, $\langle\boldsymbol{\sigma}\rangle_{M} \simeq<\boldsymbol{\sigma}>_{0}$, or at least their projection along the transformation strain. This was quite an unexpected result taking into account strong heterogeneity of all fields, significant internal stresses, and strong stress concentrators. The largest deviation from criteria (26) and (27) is due to the largest concentration with plasticity.

A larger value of PT work $W_{t}$ for the problem without plasticity compared to the problem with plasticity is connected to a larger $\mathrm{M}$ concentration. The normalized PT works in the transformed grain with plasticity and without it are plotted in Fig. 13. As can be seen, there is a linear relation with a slope of 1 between the normalized PT work $W_{t} / W_{t}^{*}$ and the concentration $c$ for both problems with plasticity in the transformed grain and without it, which confirms $\left\langle\boldsymbol{\sigma}>_{M}: \varepsilon_{t} \simeq<\boldsymbol{\sigma}>_{0}: \varepsilon_{t}\right.$ and $\left\langle\boldsymbol{\sigma}>_{M} \simeq<\boldsymbol{\sigma}>_{0}\right.$. Also, the normalized averaged PT work, $W_{t} / \Delta G \simeq c$, perfectly follows the same curve. This is true without and with plasticity in the transformed grain, however, with plasticity, there is a slight deviation from the above linear curve for case 1.

Summary. A plastic shear-induced martensitic PT starts by barrierless nucleation at the tips of dislocation pile-ups, after local stresses exceed the critical value required for the disappearance of the energy barrier. This is expected from the analytical predictions for pressureshear-induced PTs (Levitas (2004a,b)). However, after nucleation, the final stationary M morphology and concentration are governed by the local thermodynamic equilibrium at the interfaces (Eq. (23)) or thermodynamic equilibrium in terms of stresses averaged over the $\mathrm{M}$ region or entire grain (Eqs. (26) and (27)). This was not expected in the previous nanoscale models (Levitas (2004a,b); Levitas and Javanbakht (2014a)) and microscale models (Cherkaoui et al (1998); Fischer et al. (2000); Levitas et al. (1999, 2002)) for strain-induced PTs. In partic- 


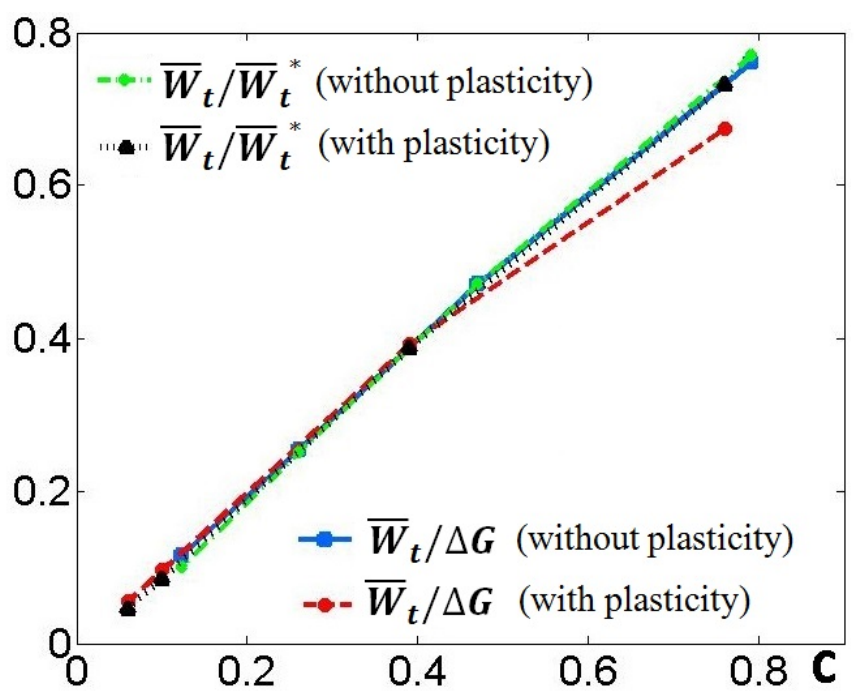

Figure 13: (color online) Normalized stationary transformation work vs. concentration with plasticity and without it.

ular, it was stated in Levitas (2004a,b) that phase equilibrium pressure (i.e., phase equilibrium conditions) does not enter the description of strain-induced PTs, which appear not to be the case at the nanoscale, both for absent and present plasticity in the transformed grain. In addition, the equality of PT work at the points of phase interface and in terms of the averaged stress tensor over the entire grain and over the $\mathrm{M}$ is also surprising since considering significant stress concentration and heterogeneity. The main difference between studies in Levitas (2004a,b) and here is that in Levitas $(2004 \mathrm{a}, \mathrm{b})$ a small single nucleus at the tip of a single dislocation pile-up was considered in an infinite space. Here, we consider a nanograined bicrystal, which can support much higher shear stresses due to the Patch-Hall effect and back stresses. The region with a large stress concentrator spreads over a large portion of transformed grain, which leads to a large transformed region (up to $c=0.79$ ), relaxing stress concentration. With allowed plasticity in the transformed grain, stress relaxed at the dislocation pile-up in the left grain but new dislocations or two new dislocation pile-ups appear within the transformed grain. All of the above differences lead to a more homogenized stress state within the transforming grain, which explains the equality of the local PT work at the equilibrium interface and two types of averaged PT work.

In addition, after PT and plastic deformation, the major part of the interfaces is dislocationfree (i.e., coherent), which corresponds to some experiments (Zhilyaev and Longdon (2000); Valiev et al. (2000)). That is why the interface equilibrium at the nanoscale is governed by 
the phase equilibrium equation, which includes transformation strain and work but does not include plastic strains. This is in contrast to the approach based on the Eshelby driving force (Cherkaoui et al (1998); Cherkaoui and Berveiller (2000); Fischer and Reisner (1998); Fischer et al. (2000)) and in accordance with the approach in Levitas (1997, 1998, 2000, 2002).

Plasticity plays a dual part in interaction with PT. Dislocation pile-ups generated during plastic flow produce strong stress tensor concentrators that lead to barrierless M nucleation. On the other hand, plasticity in the transforming grain relaxes these stress concentrators suppressing PTs. Also, some dislocations reside at the A-M interfaces, creating an athermal threshold for their propagation and arresting some parts of them.

Table 1

\begin{tabular}{|c|c|c|c|c|c|c|c|c|}
\hline & \multicolumn{4}{|c|}{ Without plasticity } & \multicolumn{4}{|c|}{ With plasticity } \\
\hline & case 1 & case 2 & case 3 & case 4 & case 1 & case 2 & case 3 & case 4 \\
\hline$c$ & 0.79 & 0.47 & 0.26 & 0.12 & 0.76 & 0.39 & 0.1 & 0.06 \\
\hline$<\sigma>_{M}: \varepsilon_{t}$ & 0.56 & 0.86 & 1.17 & 1.32 & 56 & 0.84 & 1.29 & 1.39 \\
\hline$<\boldsymbol{\sigma}>_{0}: \varepsilon_{t}$ & 0.56 & 0.86 & 1.17 & 1.32 & 52 & 0.87 & 1.31 & 1.39 \\
\hline$W_{t}$ & 0.43 & 0.40 & 29 & 0.13 & 0.38 & 0.34 & 0.11 & 0.06 \\
\hline$\Delta^{\theta} G(\theta)$ & 0.56 & 0.86 & 1.15 & 1.15 & 0.56 & 0.86 & 1.15 & 1.15 \\
\hline$W_{t}^{*}$ & 0.56 & 0.86 & 1.15 & 1.15 & 0.52 & 0.87 & 1.31 & 1.39 \\
\hline$W_{t} / \Delta^{\theta} G(\theta)$ & 0.76 & 0.48 & 0.25 & 0.12 & 0.68 & 0.39 & 0.1 & 0.06 \\
\hline$W_{t} / W_{t}^{*}$ & 0.77 & 0.47 & 0.25 & 0.10 & 0.73 & 0.39 & 0.09 & 0.05 \\
\hline
\end{tabular}

\section{Growth and arrest of a martensitic plate}

In experiments, $M$ plates grow with increasing aspect ratio until they are stopped by a strong inhomogeneity, such as a grain boundary or another M unit (Olson and Cohen (1986)). Then thickening occurs up to the equilibrium value, governed by energy of internal stresses. This is the so-called elastic growth regime. In contrast, dislocations generated by growing $\mathrm{M}$ may arrest growth within grain, leading to a lath $\mathrm{M}$ and plastic growth regime. Since microstructure determines material properties, understanding the growth problem, including plate/lath morphological transition, is of fundamental and applied importance.

The first model of the plastic growth of M plate (Olson and Cohen (1986); Haezebrouck (1987)), while based on a very simplified stress-strain fields, explained some important features, including the arrest of longitudinal plate growth because of plastic accommodation. Much more 
detailed numerical simulations of the appearance (Idesman et al. (1999)) and growth (Idesman et al. (2000); Levitas et al. (2002)) of the M unit within the A matrix based on thermodynamic criterion and kinetic equations for PT in elastoplastic materials were performed. Large strain formulation has been developed and its importance was demonstrated. This study was within a scale-free sharp interface approach and inheritance of plastic deformation was taken into account and played an important role in the growth arrest. In Cottura et al. (2012); Yeddu et al. (2012a); Malik et al. (2012); Yamanaka et al. (2008), PFA to martensitic PTs coupled to phenomenological isotropic plasticity was applied to the simulation of $\mathrm{M}$ growth. In Kundin et al. (2011a), PFA to M growth was considered with dislocations located at the moving phase interface only and dislocations did not require additional PFA equations. As we will see below, these are very restrictive assumptions, because dislocations may move away from the interface both into $A$ and $M$ regions, and they are inherited by growing phases. In Kundin et al. (2011b) plasticity during $\mathrm{M}$ growth (modeled within PFA) was described in terms of continuum dislocation theory. Plastic slip was allowed in A only and dislocations inherited by $\mathrm{M}$ kept the same eigen strain. Note that conceptually, the combination of nanoscale PFA to PT, which should resolve a nm thick phase interface, with continuum plasticity (dislocational, and even more for phenomenological), which requires the size of a representative volume from $100 \mathrm{~nm}$ to $\mathrm{mm}$, is contradictory. Also, all these approaches were for small strain approximation. In Levitas and Javanbakht (2013) a simplified PFA to coupled PTs and dislocations have been utilized for studying $\mathrm{M}$ plate growth in a single crystal. Here, a more advanced theory will be applied to elastic and plastic $\mathrm{M}$ growth in a bicrystal and new features will be determined.

\subsection{Problem formulation}

A rectangular sample with the size of $67 \times 20$ is considered which is divided into two grains with different transformation deformation gradients and different dislocation systems. The lower and upper straight sides are fixed in the $y$-direction and have zero shear stresses. The lower side can be considered as a symmetry plane; details of the boundary conditions at the upper side are not very important, since it is far from $M$ region. Restriction on strain in the vertical direction is required to arrest thickening of $\mathrm{M}$. Increase in all sizes of the system for the given problem leads to approximately proportional increase in width of $\mathrm{M}$ plates. The lateral 
sides are stress-free. Stress-free condition in the lower part of the left side is important for easy nucleation of $\mathrm{M}$ plate; this condition on the upper part of the left side can be substituted with another condition without essential effect on the solution. Stress-free conditions on the right side allow us to study interaction of PT and plasticity with the free surface, since their interaction with the grain boundary and allowing for elastic accommodation is studied while M plate propagates through the left grain. Periodic boundary conditions on the vertical sides would suppress nucleation in the left grain.

As an initial condition, a $M$ rectangular nucleus with the size of $5 \times 3$ is located at the lower left corner of the sample. Two dislocation systems inclined at $\pm 60^{\circ}$ are included in the left grain. The right grain is rotated $15^{\circ}$ counter clockwise with respect to the left grain, i.e., transformation strain is rotated and the slip systems are inclined at $+75^{\circ}$ and $-45^{\circ}$ (Fig. 14c). Initial conditions is $\xi=0.01$ for all dislocation systems. The sample is initially stress-free. A single GL equation for the same order parameters in both grains and all dislocation systems is solved.
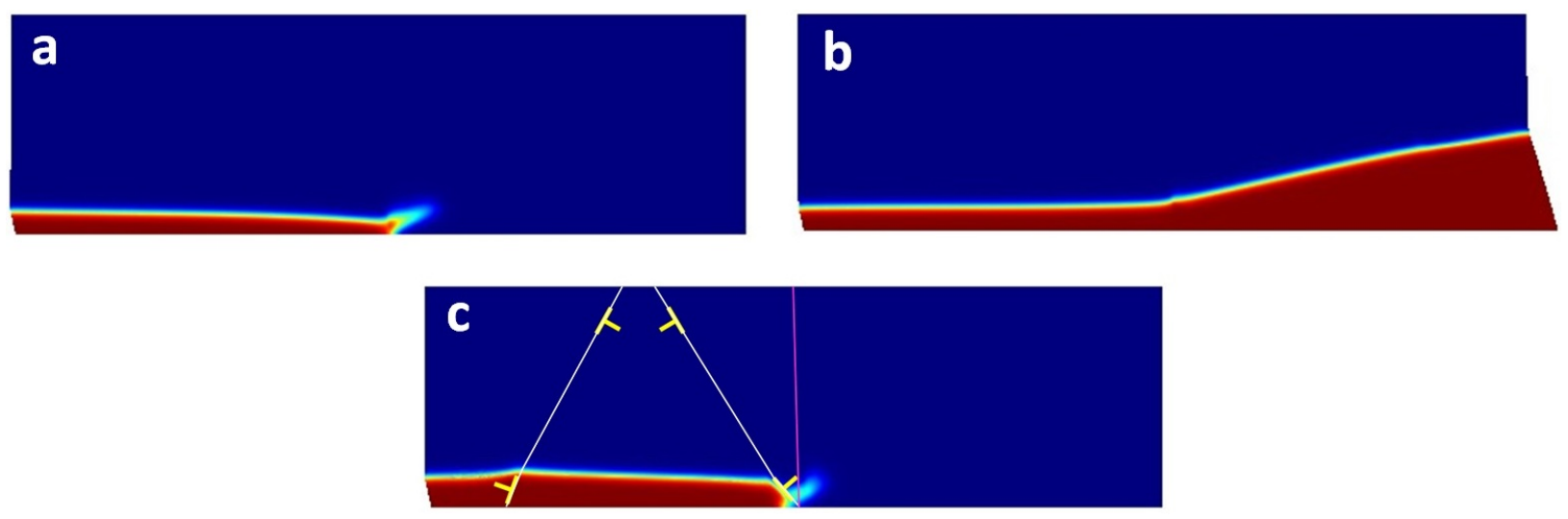

Figure 14: (color online) Stationary solutions for (a) the arrested martensitic plate at the grain boundary (without dislocations) at $\tilde{\theta}=0.417$, (b) completed martensitic plate without dislocations at $\tilde{\theta}=0.44$, and (c) arrested martensitic plate due to dislocations in the left grain at $\tilde{\theta}=0.44$.

The same material properties as in Section 3.2 are used but with $\beta_{\xi}^{A}=\beta_{\xi}^{M}=7.5 \cdot 10^{-11} N$, $A_{0}=4.4 M \mathrm{PaK}^{-1}, \beta_{\eta}^{A}=\beta_{\eta}^{M}=2.59 \cdot 10^{-10} \mathrm{~N}, L_{\eta}^{A}=L_{\eta}^{M}=2600(\mathrm{~Pa} \cdot s)^{-1}$. Also, $\varepsilon_{t}^{x}=0$, $\varepsilon_{t}^{y}=0.13$, and $\varepsilon_{t}^{x y}=-0.129$ for the left grain, and $\varepsilon_{t}^{x}=0.074, \varepsilon_{t}^{y}=0.063$, and $\varepsilon_{t}^{x y}=-0.146$ for the right grain. We introduce dimensionless temperature $\tilde{\theta}=\left(\theta_{e}-\theta\right) /\left(\theta_{e}-\theta_{c}\right)$ and the thermal driving force for PT is proportional to $\tilde{\theta}$; for phase equilibrium $\tilde{\theta}=0$; for $\mathrm{A}$ instability temperature $\theta_{c}$, one has $\tilde{\theta}=1$. 


\subsection{Phase transformation without plasticity}

Without plasticity, the $\mathrm{M}$ nucleus transforms back at $\tilde{\theta} \leq 0.39$. For $0.39<\tilde{\theta}<0.414$, the M propagates through the entire length of the left grain and creates a $\mathrm{M}$ plate, but is arrested at the boundary between two grains (Fig. 14a). For a single crystal (grain), the M plate propagates through the entire sample and then reaches an equilibrium thickness; the larger the driving force is, the larger the thickness will be. It is equilibrated by internal stresses due to transformation strain in the $y$-direction within the clamped sample. For two grains the grain boundary, due to the rotated transformation deformation gradients only, produces athermal friction and arrests the plate at a relatively small driving force. This athermal friction can be characterized in terms of $\Delta \tilde{\theta}=0.414-0.39=0.024$, overcooling temperature of $10 K$, and the critical thermal driving force $\Delta^{\theta} G(\theta)^{c}=\left(A_{0} / 3\right)\left(\theta-\theta_{e}\right)=-20 M P a$. For $\tilde{\theta} \geq 0.414$ (Fig. 14b), the $\mathrm{M}$ passes through the grain boundary and propagates through the entire right grain so that the A-M interface is inclined at $+15^{\circ}$ during propagation in the right grain (Fig. 16). This is expected, because the invariant-plane interfaces (i.e., interface with zero strains within it) are at $0^{\circ}$ in the left grain and at $15^{\circ}$ in the right grain. However, such an evolution is not always the case. Above some critical driving force, the $\mathrm{M}$ propagates differently due to a possibility of releasing larger elastic energy. For example, for $\tilde{\theta}=0.54$ the $\mathrm{M}$ does not propagate along the $15^{\circ}$ inclination in the right grain; instead after it passes through the grain boundary it propagates along the $75^{\circ}$ inclination (Fig. 15), which is not an invariant plane. In addition, $\mathrm{M}$ also nucleates from the upper right side of the right grain, and propagates along the $15^{\circ}$ inclination. As the $\mathrm{M}$ grows from the upper right side, the $\mathrm{M}$ in the middle of the sample gradually rotates from $75^{0}$ to $15^{\circ}$ (i.e., the plate at $75^{0}$ shrinks and disappears and the plate at $15^{\circ}$ grows), and then propagates through the entire right grain. At the final stage, the lower plate fills the entire lower corner and the upper region disappears. In such a way, the system significantly reduces its interface energy while producing an equilibrium concentration of $\mathrm{M}$, which is stabilized by internal stresses. The final morphology is similar to that for a smaller driving force $\tilde{\theta}$, but for a larger amount of $\mathrm{M}$.

Such a nontrivial transformation path resembles a crack branching at a large driving force. Since cracking is irreversible, the branched configuration retains. If threshold-type athermal 
friction would be introduced, such a branched $\mathrm{M}$ plate could be arrested as well. Without it the system evolves to a minimum energy configuration through a nontrivial path including direct and reverse martensitic PTs.
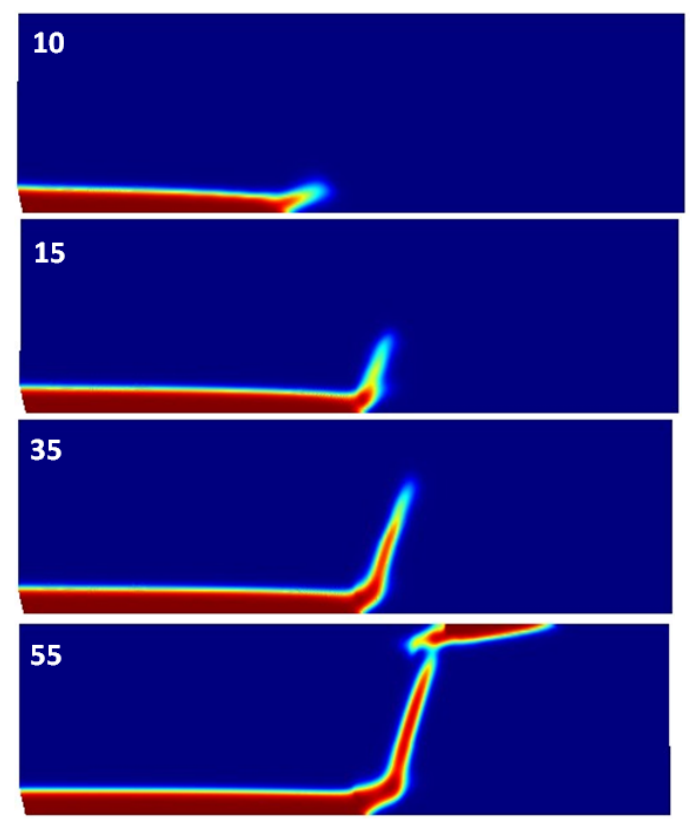
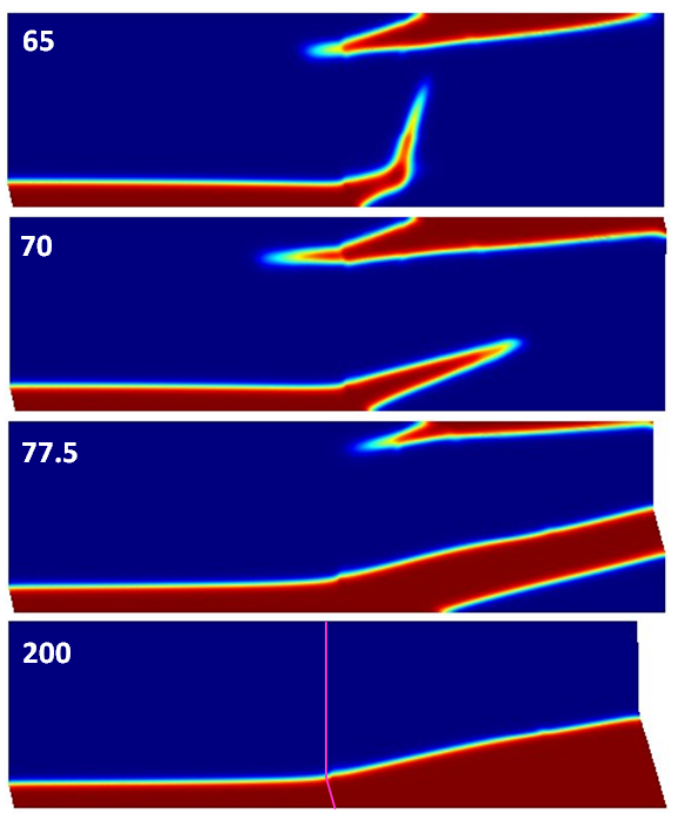

Figure 15: (color online) Evolution of martensite at $\tilde{\theta}=0.54$ without dislocations.

Note that while the kinetic relationship for the order parameter $\eta$ is linear in terms of the driving force, this results in a linear relationship between interface velocity and driving force; for each temperature an equilibrium M microstructure and concentration is reached. Change in temperature results in a fast transitional regime leading to the different stationary microstructure. For characteristic observation time exceeding such transitional regimes, kinetics looks athermal, i.e., rate-independent and depending on the driving force only. This illustrates how local rate-dependent kinetics leads to global athermal kinetics.

\subsection{Phase transformation with plasticity}

For the coupled PT and plasticity, dislocation pairs in slip plane 1 and 2 nucleate at the tip of the growing plate. Dislocations of one sign propagate toward the upper boundary of the sample and dislocations of the opposite sign remain within the M plate. For $0.414<\tilde{\theta}<0.452$, the $\mathrm{M}$ plate is arrested by one dislocation in the middle of a sample (Fig. 14c). This results in an athermal friction of $\Delta \tilde{\theta}=0.452-0.414=0.038$, or in terms of overcooling temperature 
$15 K$, and the critical thermal driving force $\Delta^{\theta} G(\theta)^{c}=\left(A_{0} / 3\right)\left(\theta-\theta_{e}\right)=-30 M P a$.

In the region of compressive stresses near the dislocation, relative thinning of the plate is observed. Since dislocations relax some internal stresses, the thickness of the plate is larger than for the elastic solution in Fig. 14b. The same is observed in a single crystal. This nanostructure remains stable up to $\tilde{\theta}=0.452$, after which growth continues through the right grain. It is also found that for $\tilde{\theta} \geq 0.452, \mathrm{M}$ is not arrested by the dislocations in the slip planes 3 and 4 in the right grain, and it grows to the right end of the sample (Fig. 16). In the slip plane 3, the dislocation pair does not appear at the tip of the plate, instead it nucleates inside the $\mathrm{M}$ plate after the $\mathrm{M}$ passes through the slip plane and reaches a certain width, and dislocations remain within the $\mathrm{M}$ plate. In the slip plane 4, first dislocation pair appears near the $M$ tip, then, with the propagation of $M$ plate, one dislocation propagates in the $A$ and is arrested at the upper sample boundary. The other dislocation passes through the $\mathrm{M}$ plate, propagates in the $\mathrm{A}$ and is arrested by the lower sample boundary. The next dislocation pairs nucleate inside the $\mathrm{M}$ plate, and the distance between dislocations of the opposite signs increases with a widening of the $\mathrm{M}$ region, and dislocations remain inside the plate or at its boundary. Compressive stresses due to extra atomic planes suppress $M$ and produces retained A. Thus, three dislocations in the low right corner of a sample are located at the A-M interface. One part of the $\mathrm{M}$ region is sheared with respect to another by the dislocations. The amount of $\mathrm{M}$ at $\tilde{\theta}=0.452$ with dislocational stress relaxation is larger than at larger driving force $\tilde{\theta}=0.54$ without dislocations.

Since slip directions (diagonals) in body (face) centric cubic and tetragonal lattices are different in the actual configuration (Fig. 1), one can see the change of slip directions across the A-M interface in Fig. 16. In some cases change in direction may be hidden due to rotation of crystal lattice across the A-M interface.

It is remarkable that most of the dislocations in the stationary solution in Fig. 16 are in $\mathrm{M}$, despite the fact that it possesses a three times larger yield strength. This, however, corresponds to experiments (Levitas et al. (2002)).

Summary. The elastic and elastoplastic growth of a $\mathrm{M}$ unit in a nano-bicrystal is studied. For elastic growth and large thermal driving force, a complex transformation path with direct and reverse PTs is observed, which still ends with the same stationary nanostructure as for 

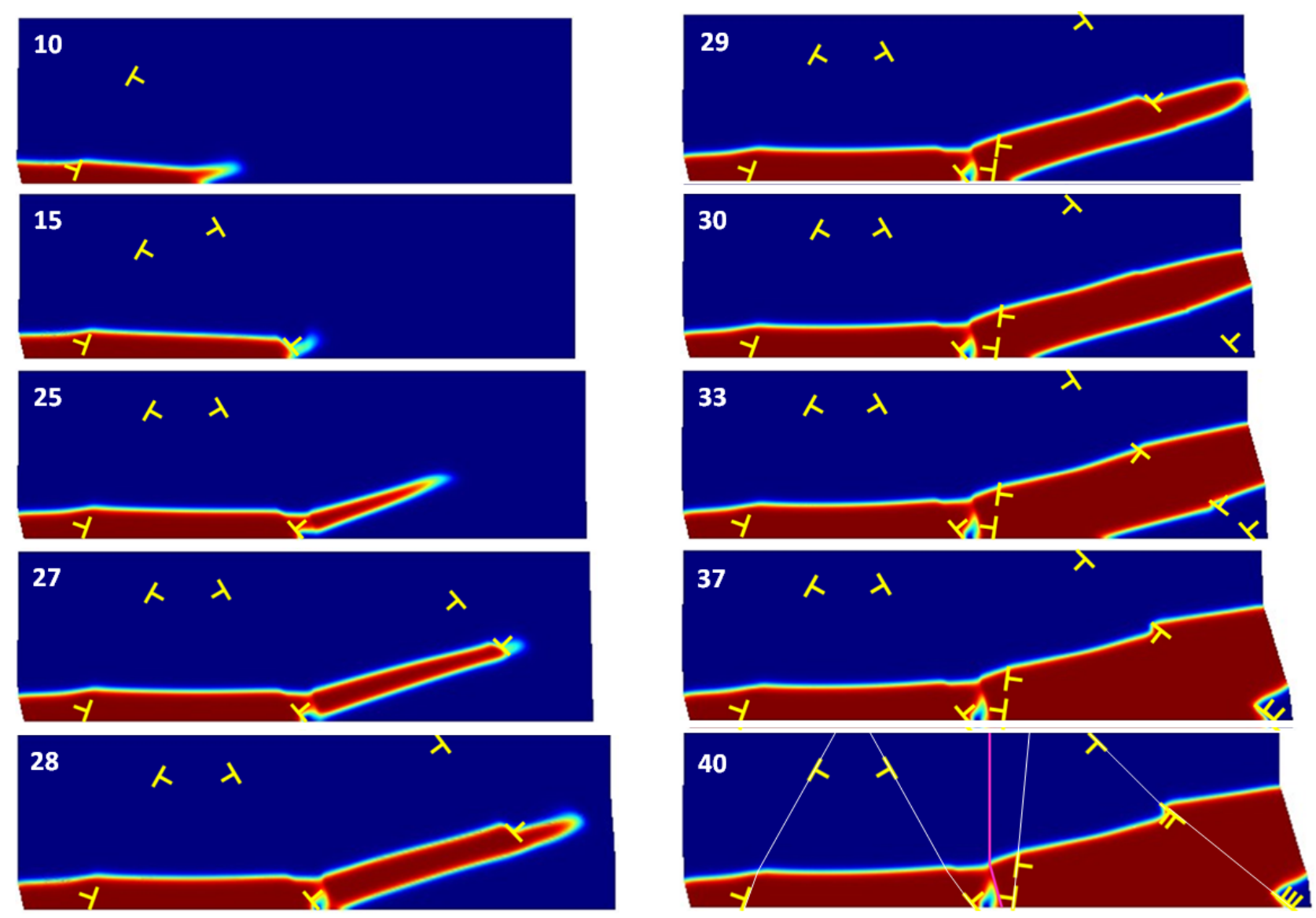

Figure 16: (color online) Evolution of martensitic plate and dislocations at $\tilde{\theta}=0.452$.

smaller driving force and traditional transformation path. Sharp grain boundary (which does not have any other properties but jump (rotation) of transformation strain) arrests plate growth at relatively small driving force, exhibiting an athermal friction. For elastoplastic growth, in addition, generation of dislocations produces athermal friction and arrests the plate as well. Still, the width of the M plate increases in comparison with elastic growth due to internal stress relaxation. When athermal friction is overcame at a lower temperature, $\mathrm{M}$ growth continues until $\mathrm{M}$ is equilibrated by internal stresses due to a clamping of the sample in $y$-direction. It is accompanied by nucleation of dislocations within $\mathrm{M}$ and remaining in $\mathrm{M}$, nucleation of dislocations at the tip of a plate and spreading them in $A$, and passing some dislocations through $M$, then through a $M-A$ interface, and then through $A$. Compressive stresses due to extra atomic planes near the dislocation pile-up suppress $M$ and shape $M$ morphology in a way that dislocations are located at A-M interface. Due to the existence of a stationary equilibrium $\mathrm{M}$ microstructure and concentration for each temperature, for large enough observation time 
one observes athermal, rate- and time-independent kinetics, even while local kinetics is based on a linear relationship between thermodynamic forces and rates. This is true for PTs and dislocations separately and when they are coupled.

Arrest of $\mathrm{M}$ by dislocation within a grain may lead to a morphological transition from plate to lath M. This transition may be utilized for controlling nanostructure and properties by controlling the dislocation mobility (Olson and Cohen (1986); Olson (1997); Levitas et al. (2002)), e.g., by alloying and preliminary plastic deformation.

\section{Concluding remarks}

In this paper, the complete system of phase field equations for coupled single-variant martensitic PT, dislocation evolution, and continuum mechanics is presented for a geometrically nonlinear case and then simplified for small strain approximation. For simplicity, we considered that the case when the slip system of the $A(M)$ transformed during a PT to $M(A)$ coincided with slip systems of $M(A)$. One needs to know dislocation core energy and width, as well as dislocation mobility in the $A$ and $M$. They vary from values in one phase to those for another phase during PT. FEM and the code COMSOL Multiphysics is utilized to solve the system of equations for two important problems. The first one is related to the simulation of shear strain-induced PT at the evolving dislocation pile-ups in a nanosized bicrystal. The dual role of the effect of plasticity on PT is found. After nucleation, the dislocations pile up near grain boundaries and produce strong stress tensor concentrators, which cause barrierless $\mathrm{M}$ nucleation. When dislocations in the transforming grain are included they relax these stress concentrators, which reduces the driving force for PT and reduces $\mathrm{M}$ concentration. The final stationary $\mathrm{M}$ morphology and concentration for both cases with and without plasticity in the transformed grain are governed by the local thermodynamic equilibrium at the interfaces. It is found that it also corresponds to the thermodynamic equilibrium in terms of the stresses averaged over the $\mathrm{M}$ region or entire grain. This is very unexpected because of previous studies Levitas (2004a,b), where it was stated that phase equilibrium conditions do not enter the macroscopic (averaged) description of strain-induced PTs. Also, the equality of PT work at the points of phase interface and in terms of averaged stresses over the $M$ and over the entire grain is very unexpected as well considering the high stress concentration and heterogeneity. The 
main reason of these differences between results in Levitas (2004a,b) and here is in different problem formulation and idealization. In Levitas (2004a,b) a small single nucleus at the tip of a single dislocation pile-up were considered in an infinite space. Here, we study a nanograined bicrystal, which can support significantly larger shear stresses due to the Patch-Hall effect and back stresses; the region with a strong stress concentrator is comparable with the entire grain, which leads to a large transformed region (up to $c=0.79$ ), relaxing stress concentration. With allowed plasticity in the transformed grain, stress relaxed at the dislocation pile-up in the left grain but new dislocations or two new dislocation pile-ups appear within transformed grain. This leads to more homogenized stress state within transforming grain and equality of the local PT work at equilibrium interface and PT work averaged over the entire grain and M.

It is found that in a stationary state the major part of the interfaces is dislocation-free (i.e., coherent), in accordance with some experiments (Zhilyaev and Longdon (2000); Valiev et al. (2000)). That is why interface equilibrium at the nanoscale is determined by PT work and does not include plastic strains. This supports the approach used in Levitas (1997, 1998, 2000, 2002) and contradicts the approach based on the Eshelby driving force (Cherkaoui et al (1998); Cherkaoui and Berveiller (2000); Fischer and Reisner (1998); Fischer et al. (2000)).

The second problem is devoted to the elastic and elastoplastic growth of the $M$ unit in a nano-bicrystal during temperature-induced PT. Two contributions to the athermal resistance to the interface motion are reproduced. The first one is due to grain boundary (in the current treatment, sharp boundary with the jump (rotation) of transformation strain) and the second one is due to the stress field of the dislocations. For elastic growth and large thermal driving force, the solution exhibits a complex transformation path with plate branching and direct and reverse PTs, which, however, evolves to the same stationary morphology as for a smaller driving force. However, if athermal resistance to the interface propagation due to the Peierls barrier and point defects would be included (e.g., like in Levitas and Lee (2007); Levitas et al. (2010)), then this intermediate configuration could be arrested, leading to a metastable microstructure. When dislocations are included in the problem (elastoplastic growth), various scenarios of their nucleation and evolution are observed. Thus, dislocations nucleate within $M$ and remain in $M$, or at the tip of a plate and spread in $A$, or pass through $M$, then $M-A$ interface, and then stop in A. As it was mentioned, dislocations produce athermal interface 
friction which arrests the $\mathrm{M}$ plate within the grain, potentially leading to a morphological transition from plate to lath M. However, they also relax internal stresses which increases the size of the stationary $\mathrm{M}$ nanostructure.

Obtained results explain how local, rate-dependent kinetics for both PTs and dislocations may lead to athermal, global, rate-independent kinetics, and how in the final nanostructure, most of the dislocations are in the $\mathrm{M}$ region despite its having a yield strength three times larger than A. Similar conclusions have been made in Levitas et al. (2002) for microscale sharpinterface study. Also, near the dislocation pile-up, the interface orientation is determined by orientation of the slip system rather than invariant-plan orientation.

A more detailed model of the grain boundaries would lead to a more precise evaluation of the corresponding athermal threshold. Also, a combination with PFA to evolve the grain structure (see, e.g., Kobavashi et al. (1998)) may lead to an additional source of stress relaxation and microstructure evolution in polycrystals. In future works, dissociation of complete dislocations into partial dislocations should be included. This, in particular, is important for dislocations inherited during PTs, if they belong to nontraditional, higher energy slip systems in the product phase lattice. Examples of treatment of dislocation reactions within PFAs are presented in Shen and Wang (2004); Hu et al. (2004); Wang et al. (2010); Hunter et al. (2011).

Microscale PFA for martemsitic PTs in elastic material suggested in Levitas et al. (2004); Idesman et al. (2005); Levitas and Ozsoy (2009a,b) can be generalized for elastoplastic materials by combining with continuum dislocation theory or crystal plasticity. Similar simulations can be produced for PFA to dislocations and reconstructive or diffusive PTs described by the Cahn-Hilliard equation.

\section{Acknowledgement}

The support of NSF (CMMI-0969143 and DMR-1434613), ARO (W911NF-12-1-0340), DARPA (W31P4Q-13-1-0010), ONR (N00014-12-1-0525), and Iowa State University are gratefully acknowledged. 
Asaro, R. J. 1983. Crystal plasticity. J. Appl. Mech. 50, 921-934.

Blank, V. D., Denisov, V. N., Kirichenko, A. N., Kulnitskiy, B. A., Martushov, S. Yu., Mavrin, B. N., Perezhogin, I. A., 2007. High pressure transformation of single-crystal graphite to form molecular carbon-onions. Nanotechnology, 18, 345601.

Boldyrev, V. V. 2006. Mechanochemistry and mechanical activation of solids. Russ. Chem. Rev. 75, 177-189.

Bridgman, P. W. 1937. Shear Phenomena at High Pressure, Particular Inorganic Compounds. Proc. Am. Acad. Arts Sci. 71, 387-460

Cherkaoui, M., Berveiller, M. 2000. Moving inelastic discontinuities and applications to martensitic phase transition. Arch. Appl. Mech. 70, 159-181.

Cherkaoui, M., Berveiller, M., Sabar, H. 1998. Micromechanical modeling of the martensitic transformation induced plasticity (TRIP) in austenitic single crystals. Int. J. Plasticity. 14, 597-626.

Cottura, M., Le Bouar, Y., Finel, A., Appolaire, B., Ammar, K., Forest, S. 2012. A phase field model incorporating strain gradient viscoplasticity: Application to rafting in Ni-base superalloys. J. Mech. Phys. Solids. A 491, 378.

Delogu, F. 2012. Are processing conditions similar in ball milling and high-pressure torsion? The case of the tetragonal-to-monoclinic phase transition in ZrO2 powders. Scripta Mater. $67,340-343$.

Edalati, K., Toh, S., Ikom, Y., Horita, Z. 2011. Plastic deformation and allotropic phase transformations in zirconia ceramics during high-pressure torsion. Scripta Mater. 974-977.

Fischer, F. D., Berveiller, M., Tanaka, K., Oberaigner, E. 1994. Continuum mechanical aspects of phase transformations in solids. Arch. Appl. Mech. 64, 54-85.

Fischer, F. D., Reisner, G. 1998. A Criterion for the martensitic transformation of a microregion in an elastic-plastic material. Acta Mater. 46, 2095-2102. 
Fischer, F. D., Reisner, G., Werner, E., Tanaka, K., Cailletaud, G., Antretter, T. 2000. A new view on transformation induced plasticity (TRIP). Int. J. Plasticity. 16, 723-748.

Fischer, F. D., Sun, Q.-P., Tanaka, K. 1996. Transformation-induced plasticity (TRIP). Appl. Mech. Rev. 49, 317-364.

Shi, J., Turteltaub, S., Van der Giessen, E. 2011. Analysis of banded morphology in multiphase steels based on a discrete dislocation transformation model. Model. Simul. Mater. Sci. Eng. $19,074006$.

Gogotsi, Y., Domnich, V. 2004. in High pressure surface science and engineering, edited by Y. Gogotsi and V. Domnich. Institute of Physics Publishing, Bristol, UK.

Green, H. W., Burnley, P. C. 1989. A new, self-organizing, mechanism for deep-focus earthquakes. Nature. 341, 773-737.

Haezebrouck, D. M., 1987. Nucleation and growth of a single martensitic particle. Doctoral thesis, Northwestern University, Materials Research Center, Steel Research Group.

Hunter, A., Beyerlein, I. J., Germann, T. C., Koslowski, M. 2011. Influence of the stacking fault energy surface on partial dislocations in fcc metals with a three-dimensional phase field dislocations dynamics model. Phys. Rev. B. 84, 144108.

Hu, S. Y., Chen, L. Q. 2001. Solute segregation and coherent nucleation and growth near a dislocation - a phase-field model integrating defect and phase microstructures. Acta. Mater. $49,463-472$.

Hu, S. Y., Chen, L. Q. 2002. Diffuse-interface modeling of composition evolution in the presence of structural defects. Comput. Mater. Sci. 23, 270-282.

Hu, S. Y., Li, Y. L., Zheng, Y. X., Chen, L. Q. 2004. Effect of solutes on dislocation motion: a phase-field simulation. Int. J. Plast. 20, 403-425.

Idesman, A. V., Levitas, V. I., Stein, E. 1997. Simulation of martensitic phase transition progress with continuous and discontinuous displacements at the interface. Comp. Mater. Sci. 9, 64-75. 
Idesman, A. V., Levitas, V. I., Stein, E. 1998. Finite element simulation of martensitic phase transitions in elastoplastic materials. Int. J. Solids Struct. 35, 855-887.

Idesman, A. V., Levitas, V. I., Stein, E. 1999. Elastoplastic materials with martensitic phase transition and twinning at finite strains: numerical solution with the finite element method. Comp. Meth. in Appl. Mech. and Eng. 173, 71-98.

Idesman, A. V., Levitas, V. I., Stein, E. 2000. Structural changes in elastoplastic materials: a unified finite element approach for phase transformation, twinning and fracture. Int. J. Plasticity. 16, 893-949.

Idesman, A. V., Levitas, V. I., Preston, D. L., Cho, J.-Y. 2005. Finite element simulations of martensitic phase transitions and microstructure based on strain softening model. J. Mech. Phys. Solids. 53, 495-523.

Ji, C., Levitas, V. I., Zhu, H., Chaudhuri, J., Marathe, A., Ma, Y. 2012. Shear-induced phase transition of nanocrystalline hexagonal boron nitride to wurtzitic structure at room temperature and lower pressure. Proc. Natl. Acad. Sci. USA. 109(47), 19108-12.

Kirby, S. W. 1987. Localized polymorphic phase transformation in high pressure faults and applications to the physical mechanism of deep focus earthquakes. J. Geophys. Res. 92, 13789-13800.

Kobayashi, R., Warren, J. A., Carter, W. C. 1998. Vector-valued phase field model for crystallization and grain boundary formation. Physica D: Nonlinear Phenomena 119, 141-150.

Koslowski, M. 2007. Scaling laws in plastic deformation. Phil. Mag., 87, 1175-1184.

Kouznetsova, V. G., Geers, M. G. D. 2008. A multi-scale model of martensitic transformation plasticity. Mech.Mater. 40, 641-657.

Kundin, J., Emmerich, H., Zimmer, J. 2011a. Mathematical concepts for the micromechanical modelling of dislocation dynamics with a phase-field approach. Philos. Mag. 91, 97-121.

Kundin, J., Raabe, D., Emmerich, H. 2011b. A phase-field model for incoherent martensitic transformations including plastic accommodation processes in the austenite. J. Mech. Phys. Solids. 59, 2082-2102. 
Lee, D.-W., Kim, H., Strachan, A., Koslowski, M., 2011. Effect of core energy on mobility in a continuum dislocation model. Phys. Rev. B. 83, 104101.

Levitas, V. I. 1995. The postulate of realizability: formulation and applications to postbifurcation behaviour and phase transitions in elastoplastic materials. Part I and II. Int. J. Eng. Sci. 33, 921-946 and 947-971.

Levitas, V. I. 1997. Phase transitions in elastoplastic materials: continuum thermomechanical theory and examples of control. Part I and II. J. Mech. Phys. Solids. 45, 923-947 and 12031222.

Levitas, V. I. 1998. Thermomechanical theory of martensitic phase transformations in inelastic materials. Int. J. Solids Struct. 35, 889-940.

Levitas, V. I. 2000. Structural changes without stable intermediate state in inelastic material. Part I and II. Int. J. Plasticity. 16, 805-849 and 851-892.

Levitas, V. I. 2002. Critical thought experiment to choose the driving force for interface propagation in inelastic materials. Int. J. Plasticity. 18, 1499-1525.

Levitas, V. I. 2004a. Continuum mechanical fundamentals of mechanochemistry. In: High Pressure Surface Science and Engineering. Section 3. Ed. Y. Gogotsi and V. Domnich, Institute of Physics Publishing. 159-292.

Levitas, V. I. 2004b. High-pressure mechanochemistry: conceptual multiscale theory and interpretation of experiments. Phys. Rev. B. 70, 184118.

Levitas, V. I. 2013b. Phase-field theory for martensitic phase transformations at large strains. Int. J. Plasticity. 49, 85-118.

Levitas, V. I., Idesman, A. V., Olson, G. B. 1999. Continuum modeling of strain-induced martensitic transformation at shear-band intersections. Acta. Mater. 47, 219-233.

Levitas, V. I., Idesman, A. V., Olson, G. B., Stein, E. 2002. Numerical modeling of martensite growth in elastoplastic material. Philos. Mag. A. 82, 429-462. 
Levitas, V. I., Idesman, A. V., Preston, D. L. 2004. Microscale simulation of evolution of martensitic microstructure. Phys. Rev. Lett. 93, 105701.

Levitas V. I., Javanbakht M. 2010. Surface tension and energy in multivariant martensitic transformations: Phase-field theory, simulations, and model of coherent interface. Phys. Rev. Lett. 105, 165701.

Levitas V. I., Javanbakht, M. 2011a. Phase-field approach to martensitic phase transformations: effect of martensite-martensite interface energy. Int. J. Mat. Research, 102, 652-665.

Levitas V. I., Javanbakht, M. 2011b. Surface-induced phase transformations: multiple scale and mechanics effects and morphological transitions. Phys. Rev. Lett. 107, 175701.

Levitas, V. I., Javanbakht, M. 2012. Advanced phase field approach to dislocation evolution. Phys. Rev. B. 86, 140101.

Levitas, V. I., Javanbakht, M. 2013. Phase field approach to interaction of phase transformation and dislocation evolution. Appl. Phys. Lett. 102, 251904.

Levitas, V.I., Javanbakht, M., 2014a. Phase transformations in nanograin materials under high pressure and plastic shear: nanoscale mechanisms. Nanoscale. 6, 162-166.

Levitas, I. V., Javanbakht, M. 2014b. Thermodynamically consistent phase field approach to dislocation evolution at large strains. J. Mech. Phys. Solids. Submitted.

Levitas, V. I., Javanbakht. M. 2015. Interaction between phase transformations and dislocations at the nanoscale. Part 1. General phase field approach. J. Mech. Phys. Solids. Submitted.

Levitas V. I., Roy A. M., Preston, D. L. 2013c. Multiple twinning and variant-variant transformations in martensite: Phase-field approach. Phys. Rev. B, 88, 054113.

Levitas V. I., Lee D. W. 2007. Athermal resistance to an interface motion in phase field theory of microstructure evolution. Phys. Rev. Lett. 99, 245701. 
Levitas, V. I., Lee, D. W., Preston, D. L. 2010. Interface propagation and microstructure evolution in phase field models of stress-induced martensitic phase transformations. Int. J. Plasticity. 26, 395-422.

Levitas, V. I., Ma, Y., Selvi, E., Wu, J., Patten J. A. 2012. High-density amorphous phase of silicon carbide obtained under large plastic shear and high pressure. Phys. Rev. B. 85, 054114 .

Levitas, V. I., Nesterenko, V. F., Meyers, M. A. Strain-induced structural changes and chemical reactions. I. Thermomechanical and kinetic models. Acta. Mater. 46, 1998, 5929-5945.

Levitas, V. I., Nesterenko, V. F., Meyers, M. A. Strain-induced structural changes and chemical reactions. II. Modelling of reactions in shear band. Acta. Mater. 46, 1998, 5947-5963.

Levitas V. I., Ozsoy I. B. 2009a. Micromechanical modeling of stress-induced phase transformations. Part 1. Thermodynamics and kinetics of coupled interface propagation and reorientation. Int. J. Plasticity. 25, 239-280.

Levitas V. I., Ozsoy I. B. 2009b. Micromechanical modeling of stress-induced phase transformations. Part 2. Computational algorithms and examples. Int. J. Plasticity. 25, 546-583.

Levitas V. I., Preston, D. L. 2002a. Three-dimensional Landau theory for multivariant stressinduced martensitic phase transformations. I. Austenite $\leftrightarrow$ Martensite. Phys. Rev. B. 66, 134206.

Levitas, V. I., Preston, D. L., 2002b. Three-dimensional Landau theory for multivariant stressinduced martensitic phase transformations. II. Multivariant phase transformations and stress space analysis. Phys. Rev. B. 66, 134207.

Levitas, V. I., Zarechnyy, O. 2010a. Modeling and simulation of strain-induced phase transformations under compression in a diamond anvil cell. Phys. Rev. B. 82, 174123.

Levitas, V. I., Zarechnyy, O. 2010b. Modeling and simulation of strain-induced phase transformations under compression and torsion in a rotational diamond anvil cell. Phys. Rev. B. 82,174124 . 
Lovey, F. C., Torra, V. 1999. Shape memory in Cu-based alloys: phenomenological behavior at the mesoscale level and interaction of martensitic transformation with structural defects

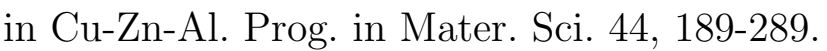

Mahnken, R., Schneidt, A., Antretter, T. 2009. Macromodelling and homogenization for transformation induced plasticity of a low-alloy steel .Int. J.Plast. 25, 183-204.

Mahnken, R., Wolff, M., Schneidt, A., Bohm, M. 2012. Multi-phase transformations at large strains - thermodynamic framework and simulation. Int. J. Plast. 39, 1-26.

Malik, A., Yeddu, H. K., Amberg, G., Borgenstam, A., Agren, J. 2012. Three dimensional elasto-plastic phase field simulation of martensitic transformation in polycrystal. Mater. Sci. Eng. A. 556, 221-232.

Marketz, F., Fischer, F. D. 1994. A micromechanical study on the coupling effect between microplastic deformation and martensitic transformation. Comput. Mater. Science. 3, 307.

Marketz, F., Fischer, F. D. 1995. A Mesoscale Study on the thermodynamic effect of stress on martensitic transformation. Metall. Mater. Trans. A. 26 A, 267-278.

Olson, G. B. 1997. Computational Design of Hierarchically Structured Materials. Science. 277, 1237-1242.

Olson, G. B., Cohen, M. 1986. Dislocation theory of martensitic transformations. In: Dislocations in Solids, Ed F. R. N., Nabarro, Amsterdam, North-Holland. 7, 297-407.

Olson, G. B., Roytburd, A. L. 1995. Martensitic nucleation. In: martensite, Eds G. B. Olson and W. S. Owen. The Materials Information Society. 9, 149-174.

Patten, J., Gao, W., Yasuto, K. 2005. Ductile Regime Nanomachining of Single-Crystal Silicon Carbide. J. Manuf. Sci. Eng. 127, 522.

Perez-Prado, M. T., Zhilyaev, A. P. 2009. First experimental observation of shear induced hcp to bcc transformation in pure Zr. Phys. Rev. Lett. 102, 175504.

Reid, A. C. E., Olson, G. B., Moran, B. 1998. Dislocations in nonlinear nonlocal media: martensitic embryo formation. Phase Transitions. 69, 309-328. 
Shen, C., Wang, Y. 2004. Incorporation of $\gamma$-surface to phase field model of dislocations: simulating dislocation dissociation in fcc crystals. Acta. Mater. 52, 683-691.

Shi, J., Turteltaub, S., Van der Giessen, E. 2010. Analysis of grain size effects on transformation-induced plasticity based on a discrete dislocationtransformation model. J. Mech. Phys. Solids. 58, 1863-1878.

Sitko, M., Skoczen, B. Effect of $\gamma-\alpha$ phase transformation on plastic adaptation to cyclic loads at cryogenic temperatures. Int. J. Solids. Struct. 49, 613-634.

Turteltaub, S., Suiker, A. S. J. 2005. Transformation-induced plasticity in ferrous alloys. J. Mech. Phys. Solids. 53, 1747-1788.

Valiev, R. Z., Islamgaliev, R. K., Alexandrov, I. V. 2000. Bulk nanostructured materials from severe plastic deformation. Prog. Mat. Sci. 45, 103-189.

Wang, Y. U., Jin, Y. M., Cuitino, A. M., Khachaturyan, A. G. 2001a. Application of phase field microelasticity theory of phase transformations to dislocation dynamics: model and three-dimensional simulations in a single crystal. Philos. Mag. 81, 385-393.

Wang, Y. U., Jin, Y. M., Cuitino, A. M., Khachaturyan, A. G. 2001b. Nanoscale phase field microelasticity theory of dislocations: model and 3D simulations. Acta Mater. 49, 1847-1857.

Wang, Y., Khachaturyan, A. G. 2006. Multi-scale phase field approach to martensitic transformations. Mater. Sci. Eng. A. 438 and 440, 55-63.

Wang, Y. U., Li, J. 2010. Phase field modeling of defects and deformation. Acta. Mater. 58, $1212-1235$.

Yamanaka, A., Takaki, T., Tomita, Y. 2008. Elastoplastic phase-field simulation of self-and plastic accommodations in cubic - tetragonal martensitic transformation. Mater. Sci. Eng. A 491,378 .

Yeddu, H. K., Borgenstam, A., Hedstram, P., Agren, J. 2012a. A phase-field study of the physical concepts of martensitic transformations in steels. Mater. Sci. Eng. A. 538, 173-181. 
Zhilyaev, A. P., Langdon, T. G. 2012. Microstructure and microtexture evolution in pure metals after ultra-high straining. J. Mater. Sci. 47, 7888-7893. 